\title{
Discontinuous Polynomial Approximations in the Theory of One-Step, Hybrid and Multistep Methods for Nonlinear Ordinary Differential Equations*
}

\author{
By M. C. Delfour and F. Dubeau
}

\begin{abstract}
This paper studies the approximation of the solution of nonlinear ordinary differential equations by (discontinuous) piecewise polynomials of degree $K$ and traces at the nodes of discretization. A mesh-dependent variational framework underlying this discontinuous approximation is derived. Several families of one-step, hybrid and multistep schemes are obtained. It is shown that the convergence rate in the $L^{2}$-norm is $K+1$. The nodal-convergence rate can go up to $2 K+2$, depending on the particular scheme under consideration. The mesh-dependent variational framework introduced here is of special interest in the approximation of the solution of optimal control problems governed by differential equations.
\end{abstract}

1. Introduction. The object of this paper is the study of (discontinuous) piecewise polynomial approximations to the solution of systems of nonlinear ordinary differential equations defined on a fixed interval $[0, T], T>0$. The type of approximation we shall use can be briefly described in the following way. The interval $[0, T]$ is first partitioned into $N$ intervals by specifying a sequence $\left\{t_{n}\right\}_{n=0}^{N}, 0=t_{0}<t_{1}<$ $\cdots<t_{N}=T$, of real numbers. On each interval $I_{n}=\left[t_{n-1}, t_{n}\right], n=1, \ldots, N$, we construct a polynomial $u_{n}$ in $P^{K}\left(I_{n}\right)$, the space of polynomials of degree at most $K \geqslant 0$ defined on the interval $I_{n}$. At each node $t_{n}$, we specify a trace (or a point) $U_{n}$, $n=0, \ldots, N$. So the approximation problem consists in finding the $N$ polynomials $\left\{u_{n}\right\}_{n=1}^{N}$ and the $N+1$ traces (or points) $\left\{U_{n}\right\}_{n=0}^{N}$. We shall see that this kind of approximation leads to a global $L^{2}$-convergence rate of $K+1$ and a nodal-convergence rate (for the traces $\left\{U_{n}\right\}$ 's) of $2 K+2$.

In this paper we adopt a more general formulation, of which the above-described approximation is a special case. On each interval $I_{n}$ we allow $L, 0 \leqslant L \leqslant K+1$, additional conditions on each polynomial $u_{n}$ in $P^{K}\left(I_{n}\right)$. For instance, when $L=2$ and $u_{n}\left(t_{n-1}\right)=U_{n-1}$ and $u_{n}\left(t_{n}\right)=U_{n}$, we obtain the continuous piecewise-polynomial approximation of B. L. Hulme [22], [23]. For that method, the global $L^{2}$-convergence rate is $K+1$ and the convergence rate at the nodes is $2 K$. This framework encompasses the $\alpha$-method of Delfour, Hager and Trochu [14] for $L=1$, and $\alpha_{n} u_{n}\left(t_{n}\right)+\left(1-\alpha_{n}\right) u_{n+1}\left(t_{n}\right)=X_{n}, n=0, \ldots, N$, where $\left\{\alpha_{n}\right\}_{n=0}^{N}$ is an a priori

Received November 30, 1981

1980 Mathematics Subject Classification. Primary 65L05.

*This research has been supported in part by Canada Natural Science and Engineering Research Council Grant A-8730, and a "Subvention FCAC du Ministère de l'Education du Québec". The second author has also been supported by a Postdoctoral Fellowship from Natural Science and Engineering Research Council. 
specified family of real numbers in the interval ]- $\infty, \frac{1}{2}$ [ (resp. $] \frac{1}{2}, \infty\left[\right.$ ) for $\alpha_{0}=0$ (resp. $\alpha_{N}=1$ ). For the $\alpha$-method, the respective global $L^{2}$-convergence and nodalconvergence rates are $K+1$ and $2 K+1$. A detailed study of this particular type of approximation can be found in [14] and will therefore not be repeated here.

We shall concentrate on families of approximation schemes which are complementary to the $\alpha$-schemes. On each interval $I_{n}$ we shall introduce $L$ conditions of the form

$$
u_{n}\left(t_{n_{l}}\right)=U_{n_{l}}, \quad l=1, \ldots, L
$$

where

$$
n_{l} \in\{n-M, n-M+1, \ldots, n\} \subset\{0,1, \ldots, N-1, N\}
$$

for some a priori fixed integer $M>0$ which is independent of both $n$ and $N$. When $L=1$, and $n_{l}=n-1$ or $n$, we obtain most of the one-step methods of the Runge-Kutta type. This situation also coincides with the $\alpha$-schemes for $\alpha_{n}=0$ and $\alpha_{n}=1$, respectively. When $L=K+1$, we obtain multistep methods. For instance, the case

$$
u_{n}\left(t_{n_{l}}\right)=U_{n-l}, \quad l=1, \ldots, K+1,
$$

leads to the the Adams-Bashforth scheme; the case

$$
u_{n}\left(t_{n_{l}}\right)=U_{n-l+1}, \quad l=1, \ldots, K+1,
$$

leads to the Adams-Moulton scheme. When $0<L<K+1$, we obtain hybrid methods. Although we shall not consider them in this paper, the present framework is also suitable for methods where on each interval $I_{n}$ the polynomial $u_{n}$ can be required to go through points ahead of $t_{n}$,

$$
\begin{gathered}
u_{n}\left(t_{n_{l}}\right)=U_{n_{l}}, \quad l=1, \ldots, L, \\
n_{l} \in\{n-M, \ldots, n, \ldots, n+M\} .
\end{gathered}
$$

It seems that convergence rates will be the same as those predicted by the theory under the more restrictive condition (1.2). The case $L=0$ first appeared in Delfour and Dubeau [13], and all the other cases are studied in F. Dubeau [17] from a slightly different point of view.

In Section 2 we shall derive the mesh-dependent variational framework underlying the discontinuous polynomial approximation schemes. In Section 3 we shall formulate the Galerkin approximation problem and prove that it always has a unique solution provided that the size of the partition

$$
h=\max \left\{t_{n}-t_{n-1}: n=1, \ldots, N\right\}
$$

is small enough and that the family of partitions is regular as $h$ goes to zero; that is, there exists $c>0$ (independent of $h$ ) such that

$$
h_{n}=t_{n}-t_{n-1} \geqslant c h, \quad n=1, \ldots, N .
$$

In Subsection 3.3 we shall give a numerical implementation of the previously mentioned methods by introducing an appropriate interpolatory quadrature formula to evaluate the integral of the nonlinear terms. In Section 4 we shall show that the respective $L^{2}$ - and nodal-convergence rates are $K+1$ and $2 K+2-L$. The general numerical scheme will be specialized to completely discontinuous methods $(L=0)$ 
in Appendix 4, to multistep methods $(L=K+1)$ in Appendix 5, to continuous methods ( $L=2, K \geqslant 1$ ) in Appendix 6 and some hybrid methods ( $L=1, \tau_{1}=1$ or 0 ) in Appendix 7. All appendices appear in the supplements section at the end of this issue. Many of these numerical schemes have been studied by different methods (cf. J. C. Butcher [4]-[8] and M. Crouzeix [11]).

The mesh-dependent variational framework underlying our discontinuous formulation can be found elsewhere for partial differential equations and boundary value problems (Babuška and Osborn [1], Babuška, Osborn and Pitkäranta [2], F. Brezzi [3], M. Fortin [18], Lesaint and Raviart [25], Oden and Wellford [28], Raviart and Thomas [29], J. M. Thomas [31], [32], Wellford and Oden [33]-[35]).

This formulation is also of the highest interest in the approximation of the solution of optimal control problems governed by differential equations. Indeed, the Galerkin approximation of the differential equation makes it possible to obtain general convergence results and to carry out the error analysis for the optimal control of the approximated problem. So the work is done only once for large families of numerical schemes. This approach can also be used to study the convergence and to do the error analysis of the matrix Riccati differential equation associated with a linear-quadratic optimal control problem (cf. J. C. Nédelec [27], M. C. Delfour [12], Delfour and Trochu [15], [16]).

Notations. $\mathbf{R}$ will denote the field of real numbers and $E$ the finite-dimensional space $\mathbf{R}^{d}$ for some integer $d \geqslant 1$. The product space in $E$ will be defined as

$$
x \cdot y=\sum_{i=1}^{d} x_{i} y_{i}, \quad x=\left(x_{1}, \ldots, x_{d}\right), y=\left(y_{1}, \ldots, y_{d}\right) \in \mathbf{R}^{d} .
$$

The following spaces of functions $f:[a, b] \rightarrow E$ will be utilized: $L^{p}([a, b] ; E)$, the space of $p$-integrable $(1 \leqslant p<\infty)$ or essentially bounded functions $(p=\infty)$, $H^{p}([a, b] ; E)$, the Sobolev space of functions with derivatives through order $p$ in $L^{2}([a, b] ; E), C([a, b] ; E)$, the space of continuous functions, $W^{1,1}([a, b] ; E)$, the space of functions in $L^{1}([a, b] ; E)$ with a first derivative in $L^{1}([a, b] ; E)$, and $P^{K}([a, b] ; E)$, the space of all polynomials of degree at most $K$. For $f \in L^{\infty}\left(I_{n} ; E\right)$, let $\|f\|_{\infty, n}$ denote the essential supremum of $|f(t)|$ over $t \in I_{n}$, where $|\cdot|$ is the Euclidean norm, and for $f \in H^{p}\left(I_{n} ; E\right)$, define

$$
\|f\|_{p, n}^{2}=\sum_{i=0}^{p}\left(f^{(i)}, f^{(i)}\right)_{n}
$$

where $(,)_{n}$ denotes the inner product in $L^{2}\left(I_{n} ; E\right)$. We also define the norms

$$
\|\cdot\|_{\infty}=\max \left\{\|\cdot\|_{\infty, n}: n=1, \ldots, N\right\} \quad \text { and } \quad\|\cdot\|_{p}^{2}=\sum_{n=1}^{N}\|\cdot\|_{p, n}^{2} .
$$

The topological dual of a real Banach space $B$ will always be written $B^{*}$.

2. Weak Forms of the Differential Equation. The object of this section is to show how a system of ordinary differential equations can be transformed into a mesh-dependent variational equation with a weak solution. This equivalent "weak formulation" provides an adequate framework for the approximation of the solution by discontinuous piecewise polynomials. 
2.1. Classical Results. Consider the following system of ordinary differential equations

$$
\dot{x}(t)=f(x(t), t) \quad \text { on }[0, T], \quad x(0)=x^{0},
$$

where $T>0$ is a real number, $x^{0} \in E$ is the initial condition, $x:[0, T] \rightarrow E$ is a vector function and $f: E \times[0, T] \rightarrow E$ is a given map. The classical existence and uniqueness results are summarized in the next theorem.

THEOREM 2.1. Assume that the following hypotheses are verified for the map $f$ :

(i) for all $x$ in $E$, the map $t \rightarrow f(x, t)$ is (Lebesgue) measurable,

(ii) there exists a function $q$ in $L^{2}(0, T ; \mathbf{R})$ such that

$$
\begin{aligned}
& \forall x_{1}, x_{2} \text { in } L^{2}(0, T ; E), \\
& \qquad\left|f\left(x_{1}(t), t\right)-f\left(x_{2}(t), t\right)\right| \leqslant q(t)\left|x_{1}(t)-x_{2}(t)\right|,
\end{aligned}
$$

(iii) the map $t \rightarrow f(0, t)$ belongs to $L^{2}(0, T ; E)$.

Then there exists a unique solution in $H^{1}(0, T ; E)$ to system (2.1).

Proof. Adaptation of the original proof of C. Carathéodory [9].

Remark 2.2. In the next subsection we shall give a proof of this theorem.

2.2. Weak Formulation. Given a partition of the interval $[0, T]$, we "transpose" the original differential equation on each subinterval of the partition. This leads to a "mesh-dependent" weak formulation of our original problem (2.1).

Definition 2.3. A partition of the interval $[0, T]$ of size $h$ is characterized by an integer $N \geqslant 1$ and a sequence $\left\{t_{n}\right\}_{n=0}^{N}$ of real numbers such that $0=t_{0}<\cdots<t_{n}$ $<\cdots<t_{N}=T$, with

$$
h=\max \left\{t_{n}-t_{n-1}: n=1, \ldots, N\right\} .
$$

Subintervals will be denoted by $I_{n}=\left[t_{n-1}, t_{n}\right], n=1, \ldots, N$.

Definition 2.4. A family of partitions indexed by $h$ is said to be regular if there exists a constant $c>0$ such that as $h$ goes to zero

$$
\forall n=1, \ldots, N, \quad c h \leqslant t_{n}-t_{n-1} \leqslant h .
$$

Throughout this paper we shall only consider regular families of partitions.

On each interval $I_{n}$, form the inner product of the first equation (2.1) with $v_{n}$ in $H^{1}\left(I_{n} ; E\right)$ and integrate by parts:

$$
x\left(t_{n}\right) \cdot v_{n}\left(t_{n}\right)=x\left(t_{n-1}\right) \cdot v_{n}\left(t_{n-1}\right)+\int_{I_{n}}\left[x \cdot \dot{v}_{n}+f(x) \cdot v_{n}\right] d t,
$$

where $f(x)$ denotes the function $t \rightarrow f(x(t), t):[0, T] \rightarrow E$. Then sum up over all $n$ equations (2.5) and the second equation (2.1) multiplied by an arbitrary $V_{0}$ in $E$. We obtain the following variational equation:

$$
\begin{aligned}
x\left(t_{0}\right) \cdot & {\left[V_{0}-v_{1}\left(t_{0}\right)\right]+\sum_{n=1}^{N-1} x\left(t_{n}\right) \cdot\left[v_{n}\left(t_{n}\right)-v_{n+1}\left(t_{n}\right)\right] } \\
& +x\left(t_{N}\right) \cdot v_{N}\left(t_{N}\right)-\sum_{n=1}^{N} \int_{I_{n}} x \cdot \dot{v}_{n} d t \\
= & x^{0} \cdot V_{0}+\sum_{n=1}^{N} \int_{I_{n}} f(x) \cdot v_{n} d t
\end{aligned}
$$


for all

$$
\tilde{v}=\left(V_{0}, v_{1}, \ldots, v_{N}\right) \in \mathscr{V}=E \times \prod_{n=1}^{N} H^{1}\left(I_{n} ; E\right) .
$$

The space $\mathscr{V}$ will be endowed with the norm

$$
\|\tilde{v}\|_{\mathscr{V}}=\left\{\left|V_{0}\right|^{2}+\sum_{n=1}^{N}\left\|v_{n}\right\|_{1, n}^{2}\right\}^{1 / 2} .
$$

This suggests the following variational problem: "To find

$$
\tilde{u}=\left(U_{0}, \ldots, U_{N}, u_{1}, \ldots, u_{N}\right) \in \mathscr{U}=E^{N+1} \times \prod_{n=1}^{N} L^{2}\left(I_{n} ; E\right)
$$

such that

$$
\begin{aligned}
U_{0} \cdot\left[V_{0}\right. & \left.-v_{1}\left(t_{0}\right)\right]+\sum_{n=1}^{N-1} U_{n} \cdot\left[v_{n}\left(t_{n}\right)-v_{n+1}\left(t_{n}\right)\right] \\
& +U_{N} \cdot v_{N}\left(t_{N}\right)-\sum_{n=1}^{N} \int_{I_{n}} u_{n} \cdot \dot{v}_{n} d t \\
= & x^{0} \cdot V_{0}+\sum_{n=1}^{N} \int_{I_{n}} f\left(u_{n}\right) \cdot v_{n} d t
\end{aligned}
$$

for all $\tilde{v}$ in $\mathscr{V} "$. Locally, this problem is completely equivalent to finding $u_{n}$ in $L^{2}\left(I_{n} ; E\right)$ and $U_{n}$ in $E$ such that

$$
\left\{\begin{array}{l}
U_{0}=x^{0} \\
U_{n} \cdot v_{n}\left(t_{n}\right)=U_{n-1} \cdot v_{n}\left(t_{n-1}\right)+\int_{I_{n}}\left[u_{n} \cdot \dot{v}_{n}+f\left(u_{n}\right) \cdot v_{n}\right] d t
\end{array}\right.
$$

for all $v_{n}$ in $H^{1}\left(I_{n} ; E\right)$, and all $n=1, \ldots, N$.

To solve (2.10), consider an elementary application of the transposition technique (Lions and Magenes [26]). This leads to the following basic result.

LEMMA 2.5. (i) For each $n$, the map

$$
v \rightarrow\left(-\dot{v}, v\left(t_{n}\right)\right): H^{1}\left(I_{n} ; E\right) \rightarrow L^{2}\left(I_{n} ; E\right) \times E
$$

is an isomorphism.

(ii) Let $b$ be an arbitrary element of $H^{1}\left(I_{n} ; E\right)^{*}$. The variational problem "to find $(u, U)$ in $L^{2}\left(I_{n} ; E\right) \times E$ such that

$$
U \cdot v\left(t_{n}\right)-\int_{I_{n}} u \cdot \dot{v}=b(v)
$$

for all $v$ in $H^{1}\left(I_{n} ; E\right)$ ", has a unique solution.

Proof. (i) Obvious. (ii) By transposition.

We can now directly solve (2.10) and in this way obtain a solution of (2.1).

THEOREM 2.6. Assume that the hypotheses of Theorem 2.1 are verified.

(i) (Existence and uniqueness). There exists a unique solution $\tilde{u}$ in $\mathscr{U}$ to the variational equation (2.10). 
(ii) (Regularity). Moreover,

$$
\tilde{u}=\left(x\left(t_{0}\right), \ldots, x\left(t_{N}\right),\left.x\right|_{I_{1}}, \ldots,\left.x\right|_{I_{N}}\right),
$$

where $x$ is the solution of problem (2.1) and $\left.x\right|_{I_{n}}$ denotes the restriction of the function $x$ to the interval $I_{n}$.

Proof. (i) (Existence and uniqueness). Notice that $U_{0}=x^{0}$. We first show that (2.10) has a unique solution if $h$ is small enough. On each interval $I_{n}$ suppose that $U_{n-1}$ is given. We then have to find $\left(u_{n}, U_{n}\right)$ in $L^{2}\left(I_{n}, E\right) \times E$ such that

$$
U_{n} \cdot v_{n}\left(t_{n}\right)-\int_{I_{n}} u_{n} \cdot \dot{v}_{n} d t=U_{n-1} \cdot v_{n}\left(t_{n-1}\right)+\int_{I_{n}} f\left(u_{n}\right) \cdot v_{n} d t
$$

If we assume that $u_{n}$ is fixed, the right-hand side of (2.13) defines an element of $H^{1}\left(I_{n} ; E\right)^{*}$. Indeed, under the hypotheses of Theorem 2.1 , and from the original work of C. Carathéodory [9], the map $t \rightarrow f\left(u_{n}(t), t\right): I_{n} \rightarrow E$ belongs to $L^{1}\left(I_{n} ; E\right)$ for all $u_{n}$ in $L^{2}\left(I_{n} ; E\right)$.

Now define the sequence $\left\{u_{n}^{i}\right\}_{i=0}^{\infty}$ as follows: $u_{n}^{0}$ is chosen arbitrarily in $L^{2}\left(I_{n} ; E\right)$, and for $i \geqslant 0, u_{n}^{i+1}$ is the solution of

$$
U_{n}^{i+1} \cdot v_{n}\left(t_{n}\right)-\int_{I_{n}} u_{n}^{i+1} \cdot \dot{v}_{n} d t=U_{n-1} \cdot v_{n}\left(t_{n-1}\right)+\int_{I_{n}} f\left(u_{n}^{i}\right) \cdot v_{n} d t
$$

for all $v_{n}$ in $H^{1}\left(I_{n} ; E\right)$. This solution is uniquely determined by Lemma 2.5 . In order to show that $\left\{u_{n}^{i}\right\}_{i=0}^{\infty}$ is a Cauchy sequence in $L^{2}\left(I_{n} ; E\right)$, subtract Eq. (2.14) from itself with $u_{n}^{i+1}$ and $u_{n}^{i}$, to obtain

$$
\begin{gathered}
{\left[U_{n}^{i+1}-U_{n}^{i}\right] \cdot v_{n}\left(t_{n}\right)-\int_{I_{n}}\left[u_{n}^{i+1}-u_{n}^{i}\right] \cdot \dot{v}_{n} d t} \\
=\int_{I_{n}}\left[f\left(u_{n}^{i}\right)-f\left(u_{n}^{i-1}\right)\right] \cdot v_{n} d t .
\end{gathered}
$$

Substitute into (2.15) the solution $v$ of the following system of equations

$$
\dot{v}(t)=u_{n}^{i}(t)-u_{n}^{i+1}(t) \text { on } I_{n}, v\left(t_{n}\right)=0 .
$$

In view of the hypotheses of Theorem 2.1, this yields

$$
\left\|u_{n}^{i+1}-u_{n}^{i}\right\|_{0, n} \leqslant h_{n}^{1 / 2}\|q\|_{0, n}\left\|u_{n}^{i}-u_{n}^{i-1}\right\|_{0, n} .
$$

Then, if $h$ is small enough, we have a contraction. Thus, $\left\{u_{n}^{i}\right\}_{i=0}^{\infty}$ is a Cauchy sequence in $L^{2}\left(I_{n} ; E\right)$ which converges to a limit $u_{n}$ in $L^{2}\left(I_{n} ; E\right)$. Moreover, by continuity, we obtain

$$
-\int_{I_{n}} u_{n} \cdot \dot{v}_{n} d t=U_{n-1} \cdot v_{n}\left(t_{n-1}\right)+\int_{I_{n}} f\left(u_{n}\right) \cdot v_{n} d t
$$

for all $v_{n}$ in $H^{1}\left(I_{n} ; E\right)$ such that $v_{n}\left(t_{n}\right)=0$. This solution is unique, as can be shown by contradiction. Finally, the vector $U_{n}$ is given by

$$
U_{n} \cdot V=U_{n-1} \cdot V+\int_{I_{n}} f\left(u_{n}\right) \cdot V d t
$$

for all $V$ in $E$, and it is clear that the pair $\left(U_{n}, u_{n}\right)$ is the unique solution of (2.13). 
We proceed in this way to the next interval and so on in a finite number of steps.

To remove the dependence on $h$, it is easy to show that the variational problem (2.10) is equivalent to "find $(u, U)$ in $L^{2}(0, T ; E) \times E$ such that

$$
U \cdot v(T)-\int_{0}^{T} u \cdot \dot{v} d t=x^{0} \cdot v(0)+\int_{0}^{T} f(u) \cdot v d t
$$

for all $v$ in $H^{1}(0, T ; E)$ ". In fact, the solutions of $(2.10)$ and (2.17) are related as follows: $U_{0}=x^{0}$ and

$$
u_{n}=\left.u\right|_{I_{n}}, \quad U_{n} \cdot V=U_{n-1} \cdot V+\int_{I_{n}} f\left(u_{n}\right) \cdot V d t
$$

for all $V$ in $E$ and $n=1, \ldots, N$.

(ii) (Regularity). Pick $v$ in $\mathscr{D}(0, T ; E)$ (the space of infinitely differentiable functions with compact support in $] 0, T[$ ). Then from (2.17) we obtain

$$
-\int_{0}^{T} u \cdot \dot{v} d t=\int_{0}^{T} f(u) \cdot v d t .
$$

Thus $u$ in $L^{2}(0, T ; E) \subset L^{1}(0, T ; E)$ has a distributional derivative in $L^{1}(0, T ; E)$ and hence belongs to $W^{1,1}(0, T ; E) \subset C(0, T ; E)$. From this fact, and hypotheses (ii) and (iii) of Theorem 2.1, we further show that the map $t \rightarrow f(u(t), t):[0, T] \rightarrow E$ belongs to $L^{2}(0, T ; E)$. Therefore $u$ belongs to $H^{1}(0, T ; E)$.

If we integrate by parts the integral term on the left-hand side of (2.17), we obtain

$$
[U-u(T)] \cdot v(T)=\left[x^{0}-u(0)\right] \cdot v(0)
$$

for all $v$ in $H^{1}(0, T ; E)$. Then $u(0)=x^{0}$ and $u(T)=U$. It is now easy to show that $U_{n}=u\left(t_{n}\right)$ for all $n$, and this proves the result.

Remark 2.7. This theorem can be generalized to solve the following variational problem: "To find $\tilde{u}$ in $\mathscr{U}$ such that

$$
\begin{aligned}
U_{0} \cdot\left[V_{0}-v_{1}\left(t_{0}\right)\right]+ & \sum_{n=1}^{N} U_{n} \cdot\left[v_{n}\left(t_{n}\right)-v_{n+1}\left(t_{n}\right)\right] \\
& +U_{N} \cdot v_{N}\left(t_{N}\right)-\sum_{n=1}^{N} \int_{I_{n}} u_{n} \cdot \dot{v}_{n} d t=b(\tilde{u})(\tilde{v})
\end{aligned}
$$

for all $\tilde{v}$ in $\mathscr{V}^{\prime \prime}$, where we consider a map $b: \mathscr{U} \rightarrow \mathscr{V}^{*}$ with the following properties:

$$
b(\tilde{u})(\tilde{v})=x^{0} \cdot V_{0}+\sum_{n=1}^{N}\left\langle b_{n}\left(u_{n}\right), v_{n}\right\rangle_{*, n},
$$

where $x^{0}$ is in $E$, and for all $n$

(i) $b_{n}: L^{2}\left(I_{n} ; E\right) \rightarrow C\left(I_{n} ; E\right)^{*}$ (the topological dual of $C\left(I_{n} ; E\right)$ ),

(ii) there exists a positive constant $\gamma$ and for every partition there exist positive constants $\left\{\gamma_{n}\right\}_{n=1}^{N}$ such that

(a) for all $u^{1}$ and $u^{2}$ in $L^{2}\left(I_{n} ; E\right)$

$$
\left\langle b_{n}\left(u^{1}\right)-b_{n}\left(u^{2}\right), v\right\rangle_{*, n} \leqslant \gamma_{n}\left\|u^{1}-u^{2}\right\|_{0, n}\|v\|_{\infty, n},
$$

(b) $\sum_{n=1}^{N} \gamma_{n}^{2} \leqslant \gamma^{2}$,

(c) $\max \left\{\gamma_{n}: n=1, \ldots, N\right\} \rightarrow 0$. 
(Here $\langle\cdot, \cdot\rangle_{*, n}$ denotes the duality product between $C\left(I_{n} ; E\right)$ and $C\left(I_{n} ; E\right)^{*}$.) In this way we can deal with systems of ordinary differential equations of the form

$$
\left\{\begin{array}{l}
\dot{x}(t)=f(x(t), t)+\sum_{j=1}^{J} \alpha_{j} \delta\left(t-t_{j}\right), \\
x(0)=x^{0}
\end{array}\right.
$$

where $0<t_{1}<\cdots<t_{J} \leqslant T$ and $\delta$ is the Dirac delta function.

3. Formulation of the Approximation Problem. In this section we use a Galerkin method to construct the approximation problem from the mesh-dependent variational equation (2.11). We then show that this approximation problem always has a unique solution for a small enough partition size $h$. Finally, the integrals of the terms containing the nonlinear function $f$ are evaluated by a specially constructed quadrature formula. This yields the general form of the numerical scheme which is ready for numerical implementation.

3.1. Galerkin Approximation Problem. Our starting point is the variational equation (2.11) defined on the spaces $\mathscr{V}$ and $\mathscr{U}$. We seek a solution $\tilde{u}^{h}$ to (2.11) in a finite-dimensional subspace $\mathscr{U}^{h}$ of $\mathscr{U}$ by requiring that $(2.11)$ be verified for all elements $\tilde{v}^{h}$ of a finite-dimensional subspace $\mathscr{V}^{h}$ of $\mathscr{V}$. This is an approximation of the Galerkin type.

Define the subspaces $\mathscr{U}^{h}$ and $\mathscr{V}^{h}$ of $\mathscr{U}$ and $\mathscr{V}$ as follows:

$$
\begin{gathered}
\mathscr{U}^{h}=\left\{\tilde{u}^{h} \mid \begin{array}{l}
\tilde{u}^{h}=\left(U_{0}, \ldots, U_{N}, u_{1}, \ldots, u_{N}\right) \in \mathscr{U} \text { such that } u_{n} \in P^{K}\left(I_{n}, E\right) \\
\text { subject to } L(\geqslant 0) \text { additional conditions for } n=1, \ldots, N
\end{array}\right\}, \\
\mathscr{V}^{h}=\left\{\tilde{v}^{h} \mid \begin{array}{l}
\tilde{v}^{h}=\left(V_{0}, v_{1}, \ldots, v_{N}\right) \in \mathscr{V} \text { such that } \\
v_{n} \in P^{K+1-L}\left(I_{n} ; E\right) \text { for } n=1, \ldots, N
\end{array}\right\},
\end{gathered}
$$

where $K$ and $L$ are positive or null integers such that $K+1-L \geqslant 0$. Note that

$$
\operatorname{dim} \mathscr{U}^{h}=[1+(K+2-L) N] \operatorname{dim} E=\operatorname{dim} \mathscr{V}^{h} .
$$

With the above choices, the approximation schemes obtained from (2.11) reduce to the following problem: "To find $\tilde{u}^{h}$ in $\mathscr{U}^{h}$ such that $U_{0}=x^{0}$ and

$$
\left\{\begin{array}{l}
U_{n} \cdot v_{n}\left(t_{n}\right)-\int_{I_{n}} u_{n} \cdot \dot{v}_{n} d t=U_{n-1} \cdot v_{n}\left(t_{n-1}\right)+\int_{I_{n}} f\left(u_{n}\right) \cdot v_{n} d t, \\
L \text { additional conditions on } u_{n}
\end{array}\right.
$$

for all $v_{n}$ in $P^{K+1-L}\left(I_{n} ; E\right)$ and $n=1, \ldots, N ”$.

We shall study the following families of approximation schemes:

(i) for $L=0$ we obtain new Galerkin methods that will be referred to as "completely discontinuous";

(ii) for $0<L \leqslant K+1$, and on each interval $I_{n}$, the $L$ conditions are of the form

$$
u_{n}\left(t_{n_{l}}\right)=U_{n}, \quad l=1, \ldots, L,
$$

where $n_{l} \in\{0, \ldots, N\}$. These Galerkin methods will be referred to as "nodal methods". To obtain existence and convergence results, we impose the following condition on the sets $\left\{n_{l}\right\}_{l=1}^{L}$ : there exists an integer $M>0$ such that for all 
$n=1, \ldots, N$ and all $l=1, \ldots, L$,

$$
n_{l} \in\{n-M, n-M+1, \ldots, n\} \text {. }
$$

Remark 3.1. The situation (ii) contains the "discontinuous Galerkin method" of Lesaint and Raviart [25] for $u_{n}\left(t_{n}\right)=U_{n}, n=1, \ldots, N$, and the "continuous Galerkin methods" of B. L. Hulme [22], [23] for $u_{n}\left(t_{n-1}\right)=U_{n-1}$ and $u_{n}\left(t_{n}\right)=U_{n}$. We also obtain multistep methods for $L=K+1$ and hybrid methods (cf. J. C. Butcher [4], C. W. Gear [19], Gragg and Stetter [20] and others) for $L<K+1$.

3.2. Solution of the Approximation Problem. We show that Eqs. (3.3) have a unique solution if $h$ is small enough. The simplest case, when $L=0$, follows directly from Subsection 2.2.

3.2.1. Completely Discontinuous Method $(L=0)$. Consider

$$
P^{K+1}\left(I_{n} ; E\right) \subset H^{1}\left(I_{n} ; E\right) \text { and } P^{K}\left(I_{n} ; E\right) \subset L^{2}\left(I_{n} ; E\right) .
$$

For the above subspaces, Lemma 2.5 and the first part of Theorem 2.6 reduce to the following lemma and theorem.

LEMMA 3.2. (i) The map

$$
v \rightarrow\left(-\dot{v}, v\left(t_{n}\right)\right): P^{K+1}\left(I_{n} ; E\right) \rightarrow P^{K}\left(I_{n} ; E\right) \times E
$$

is an isomorphism.

(ii) Let $b$ be an arbitrary element of $P^{K+1}\left(I_{n} ; E\right)^{*}$. The variational problem "to find $(u, U)$ in $P^{K}\left(I_{n} ; E\right) \times E$ such that

$$
U \cdot v\left(t_{n}\right)-\int_{I_{n}} u \cdot \dot{v}=b(v)
$$

for all $v$ in $P^{K+1}\left(I_{n} ; E\right)$ " has a unique solution.

TheOREM 3.3. Assume that the hypotheses of Theorem 2.1 are verified. The following variational problem: "To find $\tilde{u}^{h}$ in $\mathscr{U}^{h}$ such that

$$
\begin{aligned}
U_{0} \cdot\left[V_{0}-v_{1}\left(t_{0}\right)\right]+\sum_{n=1}^{N-1} U_{n} \cdot\left[v_{n}\left(t_{n}\right)-v_{n+1}\left(t_{n}\right)\right]+U_{N} \cdot v_{N}\left(t_{N}\right) \\
-\sum_{n=1}^{N} \int_{I_{n}} u_{n} \cdot \dot{v}_{n} d t=x^{0} \cdot V_{0}+\sum_{n=1}^{N} \int_{I_{n}} f\left(u_{n}\right) \cdot v_{n} d t
\end{aligned}
$$

for all $\tilde{v}$ in $\mathscr{V}^{h} "$ has a unique solution if $h$ is small enough.

3.2.2. Nodal Methods $(0<L \leqslant K+1)$. Consider the space $P^{K-L}\left(I_{n} ; E\right) \times E^{L}$ endowed with the norm

$$
\|\tilde{w}\|=\|w\|_{0, n}+h_{n}^{1 / 2} \sum_{l=1}^{L}\left|W_{l}\right|,
$$

where $w=\left(w, W_{1}, \ldots, W_{L}\right)$, and define the $L^{2}$-projector $\mathscr{P}_{L}: P^{K}\left(I_{n} ; E\right) \rightarrow$ $P^{K-L}\left(I_{n} ; E\right)$ (if $L=K+1$, set $P^{K-L}\left(I_{n} ; E\right)=\{0\}$ ).

In view of the regularity of the partitions and hypothesis (3.5) we can prove the following useful lemma. 
LEMMA 3.4. The map $J_{n}$ defined by

$$
u \rightarrow J_{n} u=\left(\mathscr{P}_{L} u, u\left(t_{n_{1}}\right), \ldots, u\left(t_{n_{l}}\right)\right): P^{K}\left(I_{n} ; E\right) \rightarrow P^{K-L}\left(I_{n} ; E\right) \times E^{L}
$$

is an isomorphism, and there exist two constants $\beta_{1}$ and $\beta_{2}$ (independent of $h$ and of the points $\left.\left\{t_{n_{l}}\right\}_{l=1}^{L}\right)$ such that

$$
\beta_{1}\|u\|_{0, n} \leqslant\left\|J_{n} u\right\| \leqslant \beta_{2}\|u\|_{0, n} .
$$

Proof. Cf. Appendix 1.

We can adapt Lemma 3.2 as follows.

LEMMA 3.5. Let $b$ be an arbitrary element of $P^{K+1-L_{L}}\left(I_{n} ; E\right)^{*}$ and let $\left\{\bar{U}_{n_{l}}\right\}_{l=1}^{L}$ be $L$ arbitrary points in $E$. The variational problem "to find $(u, U)$ in $P^{K}\left(I_{n} ; E\right) \times E$ such that

$$
U \cdot v\left(t_{n}\right)-\int_{I_{n}} u \cdot \dot{v} d t=b(v)
$$

for all $v$ in $P^{K+1-L}\left(I_{n} ; E\right)$ and

$$
u\left(t_{n_{l}}\right)=\bar{U}_{n_{1}} \text { for } l=1, \ldots, L "
$$

has a unique solution.

Proof. We solve Eqs. (3.9) and (3.10) in two steps. Firstly, apply Lemma 3.2. Then there exists $\left(u^{0}, U\right)$ in $P^{K-L}\left(I_{n} ; E\right) \times E$ such that

$$
U \cdot v\left(t_{n}\right)-\int_{I_{n}} u^{0} \cdot \dot{v} d t=b(v) .
$$

for all $v$ in $P^{K+1-L}\left(I_{n} ; E\right)$. Secondly, by Lemma 3.4 , there exists $u$ in $P^{K}\left(I_{n} ; E\right)$ such that

$$
J_{n} u=\left(u^{0}, \bar{U}_{n_{1}}, \ldots, \bar{U}_{n_{l}}\right) .
$$

Since $u^{0}$ is the $L^{2}$-projection of $u,(u, U)$ is the unique solution of (3.9) and (3.10).

THEOREM 3.6. Assume that the hypotheses of Theorem 2.1 are verified. Under conditions (3.4) and (3.5), Eqs. (3.3) have a unique solution if $h$ is small enough.

Proof. Suppose (3.3) have been solved on $I_{i}$ for $i=1, \ldots, n-1$. Define a sequence $\left\{\left(u_{n}^{i}, U_{n}^{i}\right)\right\}_{i=0}^{\infty}$ in $P^{K}\left(I_{n} ; E\right) \times E$ as follows: $\left(u_{n}^{0}, U_{n}^{0}\right)$ is chosen arbitrarily, and if $\left(u_{n}^{i}, U_{n}^{i}\right)$ is given, $\left(u_{n}^{i+1}, U_{n}^{i+1}\right)$ is the unique solution, by Lemma 3.5 , of

$$
u_{n}^{i+1}\left(t_{n_{l}}\right)= \begin{cases}U_{n_{l}} & \text { if } n_{l}<n, \\ U_{n}^{i} & \text { if } n_{l}=n,\end{cases}
$$

and

$$
-\int_{I_{n}} u_{n}^{i+1} \cdot \dot{v}_{n} d t=U_{n-1} \cdot v_{n}\left(t_{n-1}\right)+\int_{I_{n}} f\left(u_{n}^{i}\right) \cdot v_{n} d t
$$

for all $v_{n}$ in $P^{K+1-L}\left(I_{n} ; E\right)$ such that $v_{n}\left(t_{n}\right)=0$. Finally, $U_{n}^{i+1}$ is uniquely determined by

$$
U_{n}^{i+1} \cdot V=U_{n-1} \cdot V+\int_{I_{n}} f\left(u_{n}^{i+1}\right) \cdot V d t
$$

for all $V$ in $E$. 
In order to show that $\left\{u_{n}^{i}\right\}_{i=0}^{\infty}$ is a Cauchy sequence in $P^{K}\left(I_{n} ; E\right)$, subtract Eq. (3.12) from itself with $u_{n}^{i+1}$ and $u_{n}^{i}$ and set $v_{n}$ equal to the solution of the equation

$$
\dot{v}_{n}(t)=\mathscr{P}_{L}\left(u_{n}^{i}-u_{n}^{i+1}\right)(t) \quad \text { on } I_{n}, \quad v_{n}\left(t_{n}\right)=0 \text {. }
$$

This yields

$$
\left\|\mathscr{P}_{L}\left(u_{n}^{i+1}-u_{n}^{i}\right)\right\|_{0, n} \leqslant h_{n}^{1 / 2}\|q\|_{0, n}\left\|u_{n}^{i}-u_{n}^{i-1}\right\|_{0, n} .
$$

From (3.6), (3.7), (3.8) and (3.14) we have

$$
\begin{aligned}
\beta_{1}\left\|u_{n}^{i+1}-u_{n}^{i}\right\|_{0, n} \leqslant & h_{n}^{1 / 2}\|q\|_{0, n}\left\|u_{n}^{i}-u_{n}^{i-1}\right\|_{0, n} \\
& +h_{n}^{1 / 2} \sum_{l=1}^{L}\left|u_{n}^{i+1}\left(t_{n_{l}}\right)-u_{n}^{i}\left(t_{n_{l}}\right)\right| .
\end{aligned}
$$

But from (3.11) the last summation reduces at most to only one term if there exists an $l$ such that $n_{l}=n$. In this case, subtract (3.13) from itself with $U_{n}^{i}$ and $U_{n}^{i-1}$ and select $V=U_{n}^{i}-U_{n}^{i-1}$. We have

$$
\left|U_{n}^{i}-U_{n}^{i-1}\right| \leqslant\|q\|_{0, n}\left\|u_{n}^{i}-u_{n}^{i-1}\right\|_{0, n},
$$

and substitute this last result in (3.15) if necessary. Thus,

$$
\left\|u_{n}^{i+1}-u_{n}^{i}\right\|_{0, n} \leqslant \frac{2}{\beta_{1}}\|q\|_{0, n} h_{n}^{1 / 2}\left\|u_{n}^{i}-u_{n}^{i-1}\right\|_{0, n}
$$

and $\left(2 / \beta_{1}\right) h_{n}^{1 / 2}\|q\|_{0, n}$ can be made smaller than 1 for $h$ small enough. Then, $\left\{u_{n}^{i}\right\}_{i=0}^{\infty}$ is a Cauchy sequence and converges to a unique $u_{n}$ in $P^{K}\left(I_{n} ; E\right)$. Finally, select $U_{n}$ such that

$$
U_{n} \cdot V=U_{n-1} \cdot V+\int_{I_{n}} f\left(u_{n}\right) \cdot V d t
$$

for all $V$ in $E$. It is easy to show that $U_{n}^{i} \rightarrow U_{n}$ as $i \rightarrow \infty$ and $\left(u_{n}, U_{n}\right)$ is a solution of (3.3). The uniqueness follows by contradiction.

3.3. Numerical Implementation. We now turn to the problem of computing the solution of (3.3), and approximating the integral of the term which contains the nonlinear function $f$. Several examples will be given in Appendices 4-7. In order to simplify the discussion, we shall assume that $E=\mathbf{R}$ (in this section only). We shall also write $P^{K}\left(I_{n}\right)$ and $P^{K}(0,1)$ for $P^{K}\left(I_{n} ; E\right)$ and $P^{K}(0,1 ; E)$.

We first introduce a $(K+1)$-point quadrature formula which will be used in the construction of basis functions for the sets $\mathscr{U}^{h}$ and $\mathscr{V}^{h}$. We already know that on each interval $I_{n}$ the polynomial $u_{n}$ in $P^{K}\left(I_{n}\right)$ goes through the points

$$
u_{n}\left(t_{n_{l}}\right)=U_{n}, \quad l=1, \ldots, L ;
$$

moreover, we would like that the term $u_{n} \cdot \dot{v}_{n}$ (which is a polynomial of degree $2 K-L$ ) be integrated exactly.

For each $n=1, \ldots, N$ such that $0 \leqslant n_{l} \leqslant n, l=1, \ldots, L$, define the following sequence $\left\{\tau_{l}^{n}\right\}_{l=1}^{L}$ of points:

$$
\tau_{l}^{n}=\left(t_{n}-t_{n-1}\right) / h_{n}, \quad l=1, \ldots, L .
$$

In view of hypothesis (3.5) on the $n_{l}$ 's, the points $\left\{\tau_{l}^{n}\right\}_{l=1}^{L}$ all lie outside the open interval $(0,1)$. 
For simplicity, we shall assume that the sequence $\left\{\tau_{l}^{n}\right\}_{l=1}^{L}$ is independent of $n$ and denote it by $\left\{\tau_{l}\right\}_{l=1}^{L}$. However, everything that follows is true in the general case.

Consider a $(K+1)$-point quadrature formula of the form

$$
\int_{0}^{1} \psi(\tau) d \tau \simeq \sum_{k=1}^{K+1} a_{k} \psi\left(\tau_{k}\right)
$$

where the quadrature points $\left\{\tau_{k}\right\}_{k=1}^{K+1}$ are $K+1$ distinct points such that the first $L$ points $\left\{\tau_{l}\right\}_{l=1}^{L}$ are fixed outside the open interval $(0,1)$.

It is shown in Appendix 3 that we can always choose such a formula which is exact for polynomials of degree at most $2 K+1-L$; it is also shown in Appendix 3 that the remaining constructed points $\left\{\tau_{k}\right\}_{k=L+1}^{K+1}$ are distinct, unique, and all belong to the open interval $(0,1)$.

Once the quadrature points have been obtained, we use them to construct bases for $u_{n}$ in $P^{K}\left(I_{n}\right)$ and $v_{n}$ in $P^{K+1-L}\left(I_{n}\right)$. Denote by $\left\{\phi_{k}: k=1, \ldots, K+1\right\}$ the Lagrange interpolating polynomials associated with the $K+1$ points $\left\{\tau_{k}\right\}_{k=1}^{K+1}$,

$$
\phi_{k}(\tau)=\prod_{\substack{i=1 \\ i \neq k}}^{K+1} \frac{\tau-\tau_{i}}{\tau_{k}-\tau_{i}}, \quad 0 \leqslant \tau \leqslant 1, k=1, \ldots, K+1,
$$

and let

$$
\phi_{n k}(t)=\phi_{k}\left(\frac{t-t_{n-1}}{h_{n}}\right), \quad t_{n-1} \leqslant t \leqslant t_{n} .
$$

Then $\left\{\phi_{n k}: k=1, \ldots, K+1\right\}$ is the desired basis of $P^{K}\left(I_{n}\right)$, and the polynomial $u_{n}$ has the following representation

$$
u_{n}(t)=\sum_{l=1}^{L} U_{n} \phi_{n l}(t)+\sum_{k=L+1}^{K+1} u_{n k} \phi_{n k}(t)
$$

where $u_{n k}=u_{n}\left(t_{n k}\right), t_{n k}=t_{n-1}+h_{n} \tau_{k}, k=L+1, \ldots, K+1$.

The basis for the polynomials $v_{n}$ in $P^{K+1-L}\left(I_{n}\right)$ is obtained in the following manner. First, construct the Lagrange interpolating polynomials associated with the $K+1-L$ points $\left\{\tau_{k}\right\}_{k=L+1}^{K+1}$,

$$
\theta_{k}(\tau)=\prod_{\substack{i=L+1 \\ i \neq k}}^{K+1} \frac{\tau-\tau_{i}}{\tau_{k}-\tau_{i}}, \quad k=L+1, \ldots, K+1 .
$$

It forms a basis for $P^{K-L}(0,1)$. From it we construct the new family of polynomials

$$
\begin{cases}\psi_{k}(\tau)=\int_{\tau}^{1} \theta_{k}(\xi) d \xi, & 0 \leqslant \tau \leqslant 1, k=L+1, \ldots, K+1, \\ \psi_{L}(\tau)=1, & 0 \leqslant \tau \leqslant 1\end{cases}
$$

it is readily seen that the new family of polynomials $\left\{\psi_{k}: k=L, \ldots, K+1\right\}$ is a basis of $P^{K+1-L}(0,1)$. The set of polynomials

$$
\psi_{n k}(t)=\psi_{k}\left(\frac{t-t_{n-1}}{h_{n}}\right), \quad t_{n-1} \leqslant t \leqslant t_{n},
$$

will be the desired basis for $P^{K+1-L}\left(I_{n}\right)$. 
We now derive an equivalent set of equations for system (3.3). First, set $v_{n}(t)=$ $\psi_{n L}(t)=1$ in (3.3). We obtain

$$
U_{n}=U_{n-1}+\int_{I_{n}} f\left(u_{n}\right) d t
$$

Then set $v_{n}=\psi_{n k}, k=L+1, \ldots, K+1$ in Eq. (3.3) and use the quadrature (3.18) to integrate exactly the polynomial $u_{n} \cdot \dot{v}_{n}$ with $u_{n}$ given by (3.21). We obtain

$$
u_{n k}=U_{n-1}+\frac{1}{a_{k}} \sum_{l=1}^{L}\left(U_{n-1}-U_{n_{l}}\right) a_{l} \theta_{k}\left(\tau_{l}\right)+\frac{1}{a_{k}} \int_{I_{n}} f\left(u_{n}\right) \cdot \psi_{n k} d t
$$

for $k=L+1, \ldots, K+1$. This leads to the following theorem.

THEOREM 3.7. Equations (3.3) are equivalent to $U_{0}=x^{0}$ and for $n=1, \ldots, N$ such that $0 \leqslant n_{1} \leqslant n, l=1, \ldots, L$,

$$
\left\{\begin{array}{c}
u_{n}(t)=U_{n-1}+\sum_{l=1}^{L}\left(U_{n l}-U_{n-1}\right) \beta_{n l}(t) \\
\quad+\sum_{k=L+1}^{K+1} \frac{\phi_{n k}(t)}{a_{k}} \int_{I_{n}} f\left(u_{n}\right) \cdot \psi_{n k} d t \\
U_{n}=U_{n-1}+\int_{I_{n}} f\left(u_{n}\right) d t
\end{array}\right.
$$

where

$$
\beta_{n !}(t)=\phi_{n l}(t)-a_{l} \sum_{k=L+1}^{K+1} \frac{\theta_{k}\left(\tau_{l}\right)}{a_{k}} \phi_{n k}(t), \quad l=1, \ldots, L .
$$

Moreover, the $\beta_{n}$ 's belong to $P^{K}\left(I_{n}\right) \cap\left[P^{K-L}\left(I_{n}\right)\right]^{\perp} .\left(\left[P^{K-L}\left(I_{n}\right)\right]^{\perp}\right.$ denotes the orthogonal complement of $P^{K-L}\left(I_{n}\right)$ in the space $L^{2}\left(I_{n}\right)$ with respect to the inner product.)

Proof. To obtain the first equation (3.27) and (3.28), we substitute (3.26) into (3.21); the second equation (3.27) is (3.25). Finally, from (3.18) and the fact that it integrates exactly polynomials of degree less or equal to $2 K+1-L$, it is easy to show that for each $l=1, \ldots, L$,

$$
\int_{I_{n}} \beta_{n l} \theta_{n j} d t=0, \quad j=L+1, \ldots, K+1
$$

So the $\beta_{n}$ 's are orthogonal to $P^{K-L}\left(I_{n}\right)$, since the set of polynomials $\left\{\theta_{n j}: j=\right.$ $L+1, \ldots, K+1\}$ is a basis for $P^{K-L}\left(I_{n}\right)$.

COROLlary 3.8. Assume that the integral containing the nonlinear term $f$ is evaluated with the $(K+1)$-point quadrature (3.18) which is exact for polynomials of degree at most $2 K+1-L$ and which goes through the $L$ points defined by (3.17). Then Eqs. (3.3) lead to the following system of equations:

$$
U_{0}=x^{0}
$$


and for $n=1, \ldots, N$ such that $n_{l} \leqslant n, l=1, \ldots, L$,

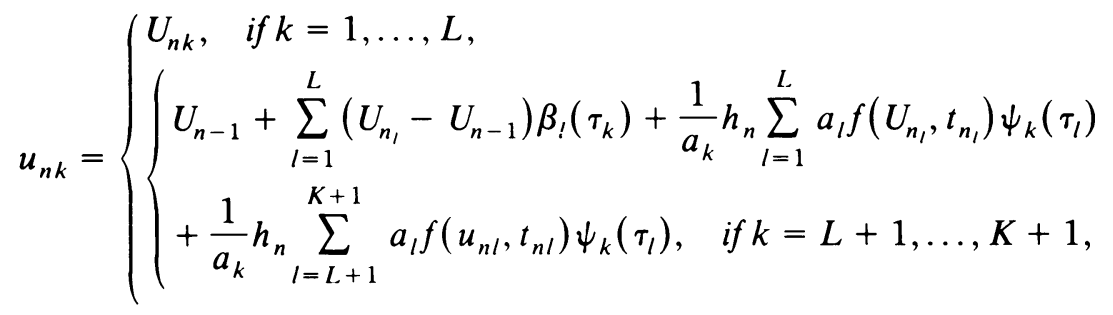

$$
U_{n}=U_{n-1}+h_{n}\left\{\sum_{l=1}^{L} a_{l} f\left(U_{n_{l}}, t_{n_{l}}\right)+\sum_{l=L+1}^{K+1} a_{l} f\left(u_{n l}, t_{n l}\right)\right\},
$$

where

$$
\beta_{l}(\tau)=\phi_{l}(\tau)-a_{l} \sum_{k=L+1}^{K+1} \frac{\theta_{k}\left(\tau_{l}\right)}{a_{k}} \phi_{k}(\tau), \quad l=1, \ldots, L .
$$

Remark 3.9. When $n_{l} \neq n, l=1, \ldots, L$, Eqs. (3.29b) and (3.29c) are uncoupled. So we have to solve a nonlinear system of $K+1-L$ equations in the $K+1-L$ variables $\left\{u_{n k}: k=L+1, \ldots, K+1\right\}$. When there exists $l$ in $\{1, \ldots, L\}$ such that $n_{1}=n$, then Eqs. (3.29b) and (3.29c) are coupled. Thence we have to solve a nonlinear system of $K+2-L$ equations in the $K+2-L$ variables $\left\{u_{n k}: k=L+1, \ldots, K+1\right\} \cup\left\{U_{n}\right\}$. This can be done by iteration.

Remark 3.10. The various coefficients in Eq. (3.29b) can be easily computed from the quadrature points $\left\{\tau_{k}\right\}_{k=1}^{K+1}$ and weights $\left\{a_{k}\right\}_{k=1}^{K+1}$. Moreover,

$$
\beta_{l}\left(\tau_{k}\right)=-\theta_{k}\left(\tau_{l}\right) a_{l} / a_{k}, \quad l=1, \ldots, L, k=L+1, \ldots, K+1 .
$$

COROLlaRY 3.11. The polynomials $\beta_{l}, l=1, \ldots, L$, can be computed by the procedure of Appendix 3 in the following manner. For a fixed l, the polynomial $\beta_{l}$ is the product of two polynomials $\Omega$, and $\omega_{1}$ of the following form:

$$
\begin{gathered}
\Omega_{l}(\tau)=\prod_{\substack{j=1 \\
j \neq l}}^{L} \frac{\tau-\tau_{j}}{\tau_{l}-\tau_{j}} \text { in } P^{L-1}(0,1), \\
\omega_{l}(\tau)=\prod_{k=L+1}^{K} \frac{\tau-\bar{\tau}_{k}}{\tau_{l}-\bar{\tau}_{k}} \frac{\tau-\bar{\tau}_{l}}{\tau_{l}-\bar{\tau}_{l}} \quad \text { in } P^{K+1-L}(0,1),
\end{gathered}
$$

where the points $\left\{\bar{\tau}_{l}\right\} \cup\left\{\bar{\tau}_{k}: k=L+1, \ldots, K\right\}$ can be uniquely chosen in such a way that the polynomial $\omega_{1} \Omega$, be orthogonal to the space $P^{K-L}(0,1)$ on the interval $(0,1)$.

Proof. Cf. Appendix 3 with $L$ and $K$ replaced by $L-1$ and $K-1$ and the set $\left\{\tau_{k}: k=1, \ldots, L\right\}$ replaced by the set $\left\{\tau_{k}: k=1, \ldots, L, k \neq l\right\}$.

Remark 3.12. We shall see in Section 4 that the predicted $L^{2}$ - and nodal-convergence rates are $K+1$ and $2 K+2-L$, respectively. The use of a quadrature formula which integrates exactly polynomials of degree $2 K+1-L$ will not affect those rates (cf. F. Dubeau [17]).

The integral of the term containing the nonlinear function $f$ can also be evaluated by an arbitrary $J$-point quadrature formula of the form

$$
\int_{0}^{1} \psi(\xi) d \xi \simeq \sum_{j=1}^{J} w_{j} \psi\left(\xi_{j}\right)
$$


where $\left\{\xi_{j}: j=1, \ldots, J\right\}$ are $J$ distinct real numbers. On the interval $I_{n},(3.34)$ becomes

$$
\int_{I_{n}} g(t) d t \simeq h_{n} \sum_{j=1}^{J} w_{j} g\left(t_{n j}\right)
$$

where $t_{n j}=t_{n-1}+h_{n} \xi_{j}, j=1, \ldots, J$.

Corollary 3.13. Assume that the integral of the term containing the nonlinear function $f$ is evaluated by (3.35). Then Eqs. (3.3) yield

$$
U_{0}=x^{0}
$$

and for $n=1, \ldots, N$ such that $n_{l} \leqslant n, l=1, \ldots, L$, and for $i=1, \ldots, J$,

$$
\begin{aligned}
\bar{u}_{n i}= & U_{n-1}+h_{n} \sum_{j=1}^{J} w_{j} f\left(\bar{u}_{n j}\right)\left[\sum_{k=L+1}^{K+1} \frac{\phi_{k}\left(\xi_{i}\right)}{a_{k}} \psi_{k}\left(\xi_{j}\right)\right] \\
& +\sum_{\substack{l=1 \\
n \neq \neq}}^{L}\left(U_{n_{l}}-U_{n-1}\right) \beta_{l}\left(\xi_{i}\right) \\
& +\left\{\begin{array}{l}
h_{n} \beta_{m}\left(\xi_{i}\right) \sum_{j=1}^{J} w_{j} f\left(\bar{u}_{n_{j}}\right), \quad \text { if } \exists m \in\{1, \ldots, L\} \text { s.t. } n_{m}=n, \\
0, \text { otherwise, }
\end{array}\right.
\end{aligned}
$$

$$
U_{n}=U_{n-1}+h_{n} \sum_{j=1}^{J} w_{j} f\left(\bar{u}_{n j}\right)
$$

where for $j=1, \ldots, J$,

$$
\bar{t}_{n j}=t_{n-1}+h_{n} \xi_{j}, \quad \bar{u}_{n j}=u_{n}\left(\bar{t}_{n j}\right) \quad \text { and } f\left(\bar{u}_{n j}\right)=f\left(u_{n}\left(\bar{t}_{n j}\right), \bar{t}_{n j}\right) .
$$

Moreover, Eq. (3.26) becomes

$$
u_{n k}=U_{n-1}+\frac{1}{a_{k}} \sum_{l=1}^{L}\left(U_{n-1}-U_{n_{l}}\right) a_{l} \theta_{k}\left(\tau_{l}\right)+\frac{h_{n}}{a_{k}} \sum_{j=1}^{J} w_{j} f\left(\bar{u}_{n j}\right) \psi_{k}\left(\xi_{j}\right),
$$

$k=L+1, \ldots, K+1$, and $u_{n}$ is determined by (3.21).

Remark 3.14. Again, if formula (3.34) integrates exactly polynomials of degree $2 K+1-L$, the convergence rates of (3.29) are preserved for (3.36) (cf. F. Dubeau [17]).

4. Error Estimates. This section contains the main two theorems which establish the convergence and provide asymptotic error estimates. The first theorem shows that if the solution of $(2.1)$ belongs to $H^{K+1}(0, T ; E)$, the $L^{2}$ and nodal errors are proportional to $h^{K+1}$. The second theorem shows that under appropriate hypotheses on the function $f$ there is an asymptotic superconvergence at the nodes proportional to $h^{2 K+2-L}$ (recall that $0 \leqslant L \leqslant K+1$ ).

Throughout this section we assume that $f$ satisfies the hypotheses of Theorem 2.1, that the family of partitions is regular as its size $h$ goes to zero, and that $h$ is sufficiently small for the existence of a unique solution $\tilde{u}$ to (3.3). The letter $c$ will denote a generic constant independent of $h$. 
4.1. $L^{2}$ and Nodal Errors.

TheOREM 4.1. Assume that the solution $x$ of $(2.1)$ belongs to $H^{K+1}(0, T ; E)$. For $M>1$, assume that on the first $M-1$ intervals the solution $\tilde{u}$ of $(3.3)$ is such that

$$
\max \left\{\left|U_{n}-x\left(t_{n}\right)\right|: n=0, \ldots, M-1\right\} \leqslant c h^{K+1}\left\|x^{(K+1)}\right\|_{0}
$$

and for $j=0, \ldots, K+1$,

$$
\left\{\sum_{n=1}^{M-1}\left\|u_{n}-x\right\|_{j, n}^{2}\right\}^{1 / 2} \leqslant c h^{K+1-j}\left\|x^{(K+1)}\right\|_{0}
$$

(when $M=1$, we use the fact that $\left|U_{0}-x\left(t_{0}\right)\right|=0$ ), where $x^{(K+1)}$ denotes the $(K+1)$ st derivative of $x$. Then, when $h$ is small enough,

$$
\max \left\{\left|U_{n}-x\left(t_{n}\right)\right|: n=0, \ldots, N\right\} \leqslant c h^{K+1}\left\|x^{(K+1)}\right\|_{0},
$$

and for $j=0, \ldots, K+1$,

$$
\left\|u^{h}-x\right\|_{j} \leqslant c h^{K+1-j}\left\|x^{(K+1)}\right\|_{0},
$$

where

$$
u^{h}=\sum_{n=1}^{N} u_{n} \chi_{I_{n}} \text { and }\|\cdot\|_{j}=\left\{\sum_{n=1}^{N}\|\cdot\|_{j, n}^{2}\right\}^{1 / 2}
$$

$\left(\chi_{I_{n}}\right.$ is the characteristic function of $\left.I_{n}\right)$.

Remark 4.2. Inequalities (4.1) and (4.2) are the standard hypotheses in the analysis of multistep methods.

Proof of Theorem 4.1. Since $x$ verifies Eqs. (3.3), we have

$$
\begin{aligned}
{\left[U_{n}-x\left(t_{n}\right)\right] \cdot v_{n}\left(t_{n}\right)=} & {\left[U_{n-1}-x\left(t_{n-1}\right)\right] \cdot v_{n}\left(t_{n-1}\right)+\int_{I_{n}}\left[u_{n}-x\right] \cdot \dot{v}_{n} d t } \\
& +\int_{I_{n}}\left[f\left(u_{n}\right)-f(x)\right] \cdot v_{n} d t .
\end{aligned}
$$

Substitute $v_{n}=U_{n}-x\left(t_{n}\right)$ into (4.5):

$$
\left|U_{n}-x\left(t_{n}\right)\right| \leqslant\left|U_{n-1}-x\left(t_{n-1}\right)\right|+\|q\|_{0, n}\left\|u_{n}-x\right\|_{0, n} .
$$

Now introduce an arbitrary polynomial $\bar{u}_{n}$ in $P^{K}\left(I_{n} ; E\right)$. We seek a bound on the term $\left\|\mathscr{P}_{L}\left(u_{n}-\bar{u}_{n}\right)\right\|_{0, n}$, where $\mathscr{P}_{L}$ is the $L^{2}$-projector of $L^{2}\left(I_{n} ; E\right)$ onto $P^{K-L}\left(I_{n} ; E\right)$. Substitute for $v_{n}$ into $(4.5)$ the solution of the equation

$$
\dot{v}_{n}(t)=-\mathscr{P}_{L}\left(u_{n}-\bar{u}_{n}\right)(t), \quad t \in I_{n}, \quad v_{n}\left(t_{n}\right)=0 .
$$

We obtain the following first estimate

$$
\begin{aligned}
\left\|\mathscr{P}_{L}\left(u_{n}-\bar{u}_{n}\right)\right\|_{0, n} \leqslant & h_{n}^{1 / 2}\left|U_{n-1}-x\left(t_{n-1}\right)\right|+h_{n}^{1 / 2}\|q\|_{0, n}\left\|u_{n}-\bar{u}_{n}\right\|_{0, n} \\
& +\left[1+h_{n}^{1 / 2}\|q\|_{0, n}\right]\left\|\bar{u}_{n}-x\right\|_{0, n} .
\end{aligned}
$$


From (3.8) and the last inequality we obtain

$$
\begin{aligned}
{\left[\beta_{1}-\right.} & \left.h_{n}^{1 / 2}\|q\|_{0, n}\right]\left\|u_{n}-\bar{u}_{n}\right\|_{0, n} \\
\leqslant & h_{n}^{1 / 2}\left|U_{n-1}-x\left(t_{n-1}\right)\right|+\left[1+h_{n}^{1 / 2}\|q\|_{0, n}\right]\left\|\bar{u}_{n}-x\right\|_{0, n} \\
& +h_{n}^{1 / 2} \sum_{l=1}^{L}\left|u_{n}\left(t_{n_{l}}\right)-\bar{u}_{n}\left(t_{n_{l}}\right)\right| .
\end{aligned}
$$

If $h$ is small enough, (4.6) and (4.7) yield

$$
\begin{aligned}
\left|U_{n}-x\left(t_{n}\right)\right| \leqslant & \left|U_{n-1}-x\left(t_{n-1}\right)\right| \\
& +c h_{n}^{1 / 2}\|q\|_{0, n}\left[\left|U_{n-1}-x\left(t_{n-1}\right)\right|+\sum_{l=1}^{L}\left|u_{n}\left(t_{n_{l}}\right)-\bar{u}_{n}\left(t_{n_{l}}\right)\right|\right] \\
& +c\|q\|_{0, n}\left\|\bar{u}_{n}-x\right\|_{0, n} .
\end{aligned}
$$

From (4.7), we also have

$$
\begin{aligned}
\left\|u_{n}-x\right\|_{0, n} \leqslant & \operatorname{ch}_{n}^{1 / 2}\left[\left|U_{n-1}-x\left(t_{n-1}\right)\right|+\sum_{l=1}^{L}\left|u_{n}\left(t_{n_{l}}\right)-\bar{u}_{n}\left(t_{n_{l}}\right)\right|\right] \\
& +c\left\|\bar{u}_{n}-x\right\|_{0, n} .
\end{aligned}
$$

Now if $\bar{u}_{n}$ is the Lagrange interpolating polynomial of degree $K$ such that

$$
\bar{u}_{n}\left(t_{n}\right)=x\left(t_{n_{l}}\right), \quad l=1, \ldots, L \leqslant(K+1),
$$

we can use the following result of interpolation theory:

LEMMA 4.3. Let $P$ be a linear operator from $H^{K+1}(I ; E)$ to $H^{J}(I ; E)$ such that $P u=u$ for all $u$ in $P^{K}(I ; E)$ where $0 \leqslant J \leqslant K+1$. Then there exists a constant $c$ independent of $\operatorname{mes}(I)$ such that for every $x$ in $H^{K+1}(I ; E)$ we have

$$
\|P x-x\|_{J} \leqslant c(\operatorname{mes} I)^{K+1-J}\left\|x^{(K+1)}\right\|_{0} .
$$

Proof. Cf. P. G. Ciarlet [10] or Strang and Fix [30].

Since $\left[t_{n-1}, t_{n}\right] \subset I=\left[t_{n-M}, t_{n}\right]$ and mes $I \leqslant M h$ (cf. hypothesis (3.5)), we obtain from Lemma 4.3

$$
\left\|\bar{u}_{n}-x\right\|_{0, n} \leqslant\left\|\bar{u}_{n}-x\right\|_{0, \bar{n}} \leqslant c(\operatorname{mes} I)^{K+1}\left\|x^{(K+1)}\right\|_{0, \bar{n}} \leqslant c^{\prime} h^{K+1}\left\|x^{(K+1)}\right\|_{0, \bar{n}},
$$

where $\|\cdot\|_{0, \bar{n}}$ is the $L^{2}$-norm over $I$. Also noting that $L \leqslant M$ and that $u\left(t_{n_{l}}\right)=U_{n l}$, inequality (4.8) can be rearranged in the form

$$
\begin{aligned}
& \left(1-c\|q\|_{0, n} h_{n}^{1 / 2}\right)\left|U_{n}-x\left(t_{n}\right)\right| \\
& \quad \leqslant\left|U_{n-1}-x\left(t_{n-1}\right)\right|+c h_{n}^{1 / 2}\|q\|_{0, n} \sum_{i=1}^{M}\left|U_{n-i}-x\left(t_{n-i}\right)\right|+c h^{K+1}\left\|x^{(K+1)}\right\|_{0, \bar{n}} .
\end{aligned}
$$

Finally, for some constant $c>0$, inequalities (4.8) and (4.9) become

$$
\begin{aligned}
\left|U_{n}-x\left(t_{n}\right)\right| \leqslant & \left|U_{n-1}-x\left(t_{n-1}\right)\right|+\operatorname{ch}_{n}^{1 / 2}\|q\|_{0, n} \sum_{i=1}^{M}\left|U_{n-i}-x\left(t_{n-i}\right)\right| \\
& +\operatorname{ch}^{K+1}\|q\|_{0, n}\left\|x^{(K+1)}\right\|_{0, \bar{n}}, \\
\left\|u_{n}-x\right\|_{0, n} \leqslant & \operatorname{ch}_{n}^{1 / 2} \sum_{i=0}^{M}\left|U_{n-i}-x\left(t_{n-i}\right)\right|+\operatorname{ch}^{K+1}\left\|x^{(K+1)}\right\|_{0, \bar{n}} .
\end{aligned}
$$


Now Lemma A2.2 of Appendix 2 can be applied since inequality (4.10) is of the same form as inequality (3) in Appendix 2. So using the estimates

$$
\begin{aligned}
& \prod_{n=1}^{N}\left(1+c M h_{n}^{1 / 2}\|q\|_{0, n}\right) \leqslant \exp \left(c M T^{1 / 2}\|q\|_{0}\right) \\
& \sum_{n=M}^{N}\|q\|_{0, n}\left\|x^{(K+1)}\right\|_{0, \bar{n}} \leqslant\|q\|_{0}\left\{\sum_{n=M}^{N}\left\|x^{(K+1)}\right\|_{0, \bar{n}}^{2}\right\} \leqslant M^{1 / 2}\|q\|_{0}\left\|x^{(K+1)}\right\|_{0},
\end{aligned}
$$

we obtain

$$
\left|U_{n}-x\left(t_{n}\right)\right| \leqslant c\left[\max \left\{\left|U_{i}-x\left(t_{i}\right)\right|: i=0, \ldots, M-1\right\}+h^{K+1}\left\|x^{(K+1)}\right\|_{0}\right] .
$$

In the case $M=1,(4.1)$ is trivially verified, since $\left|U_{0}-x\left(t_{0}\right)\right|=0$ and (4.2) does not enter into consideration. Then (4.3) follows directly from (4.1), and (4.4) with $j=0$ from (4.11) and (4.3). Inequalities (4.4) for $1 \leqslant j \leqslant K+1$ are obtained by using the inequalities

$$
\left\|u_{n}-x\right\|_{j, n} \leqslant\left\|u_{n}-\bar{u}_{n}\right\|_{j, n}+\left\|\bar{u}_{n}-x\right\|_{j, n}
$$

and

$$
\left\|u_{n}-\bar{u}_{n}\right\|_{j, n} \leqslant c h^{-j}\left\|u_{n}-\bar{u}_{n}\right\|_{0, n} .
$$

The final result follows from (4.7), (4.2) and (4.3).

Remark 4.4. The smoothness of $x$ is useful only when we apply an interpolatory result. For the case $L=0$, without any smoothness hypothesis on $x$, we obtain

$$
\left\|u^{h}-x\right\|_{0} \leqslant c \inf \left\{\left\|\bar{u}^{h}-x\right\|_{0}: \bar{u}_{n}=\left.\bar{u}^{h}\right|_{I_{n}} \in P^{K}\left(I_{n} ; E\right) \text { for } n=1, \ldots, N\right\} \text {. }
$$

In some situations, this might be useful to obtain simple convergence.

COROLLARY 4.5. Under the hypotheses of Theorem 4.1, we have

$$
\left\|u_{n}-x\right\|_{\infty, n} \leqslant c h^{K+1 / 2}\left\|x^{(K+1)}\right\|_{0}
$$

for all $n=1, \ldots, N$.

Proof. Use the following inequality:

$$
\left\|u_{n}-x\right\|_{\infty, n} \leqslant h_{n}^{-1 / 2}\left[\left\|u_{n}-x\right\|_{0, n}+h_{n}\left\|u_{n}-x\right\|_{1, n}\right] .
$$

The result follows from (4.4).

4.2. Superconvergence Results. Under additional hypotheses we can improve the rate of convergence at the nodes.

TheOREM 4.6. Assume that the hypotheses of Theorem 4.1 hold. Assume also that

(i) the matrix

$$
A(t)=f_{x}(x(t), t) \quad\left(a_{i j}=\frac{\partial f_{i}}{\partial x_{j}}(x(t), t)\right)
$$

exists and that its columns belong to $H^{K+1-L}(0, T ; E)$, and that

(ii) there exists a neighborhood $V$ of the origin in $E$ and a positive constant $B$ such that for all $t$ and all $y$ in $x(t)+V$,

$$
|f(y, t)-f(x(t), t)-A(t)(y-x(t))| \leqslant B|x(t)-y|^{2} .
$$


Then for $h$ small enough,

(4.14) $\max \left\{\left|U_{n}-x\left(t_{n}\right)\right|: n=0, \ldots, N\right\} \leqslant c\left\|u^{h}-x\right\|_{0}\left[h^{K+1-L}+\left\|u^{h}-x\right\|_{0}\right]$.

Proof. Choose $\tilde{v}=\left(V_{0}, v_{1}, \ldots, v_{N}\right)$ such that $v_{1}\left(t_{0}\right)=V_{0}$ and $v_{n}\left(t_{n}\right)=v_{n+1}\left(t_{n}\right)$, $n=1, \ldots, N-1$. Substitute that $\tilde{v}$ in (4.5) and sum up over $j=1, \ldots, n$. This yields

$$
\left[U_{n}-x\left(t_{n}\right)\right] \cdot v_{n}\left(t_{n}\right)=\int_{0}^{t_{n}}\left[u^{h}-x\right] \cdot \dot{v}^{h} d t+\int_{0}^{t_{n}}\left[f\left(u^{h}\right)-f(x)\right] \cdot v^{h} d t .
$$

Let $w$ in $H^{K+2-L}(0, T ; E)$ be the solution of

$$
\dot{w}(t)+A^{T} w(t)=0 \quad \text { on }[0, T], \quad w\left(t_{n}\right)=U_{n}-x\left(t_{n}\right),
$$

and let $w^{h}$ be a continuous piecewise interpolating polynomial of degree $K+1-L$ of $w$ such that $w^{h}\left(t_{n}\right)=U_{n}-x\left(t_{n}\right)$. From Lemma 4.3,

$$
\left\|w-w^{h}\right\|_{1} \leqslant c h^{K+1-L}\left\|w^{(K+2-L)}\right\|_{0} .
$$

Now use the following lemma to express the norm of $w^{(K+2-L)}$ in terms of $U_{n}-x\left(t_{n}\right)$.

LeMma 4.7. Fix $s$ in $[0, T]$. Suppose that $g$ is such that $\left.g\right|_{I_{n}} \in H^{K+1-L}\left(I_{n} ; E\right)$ for $n=1, \ldots, N$, and that $w$ in $H^{1}(0, T ; E)$ satisfies the equation

$$
\dot{w}(t)+A^{T}(t) w(t)=g(t) \quad \text { on }[0, T] .
$$

Then there exists a constant $c$ independent of $g$ and $s \in[0, T]$ such that

$$
\|w\|_{K+2-L} \leqslant c\left\{|w(s)|+\|g\|_{K+1-L}\right\} \text {. }
$$

(Note that $\left.w\right|_{I_{n}}$ belongs to $H^{K+2-L}\left(I_{n} ; E\right)$.)

Proof. Cf. Delfour, Hager, and Trochu [14].

We then have

$$
\left\|w-w^{h}\right\|_{1} \leqslant c h^{K+1-L}\left|U_{n}-x\left(t_{n}\right)\right| .
$$

Set $v^{h}=w^{h}$ in (4.15). By Corollary 4.5, $u_{n}(t) \in x(t)+V$ for $h$ small enough. Now we can use (4.13) to obtain

$$
\left|U_{n}-x\left(t_{n}\right)\right|^{2} \leqslant\left\|u^{h}-x\right\|_{0}\left\|\dot{w}^{h}+A^{T} w^{h}\right\|_{0}+B\left\|u^{h}-x\right\|_{0}^{2}\left\|w^{h}\right\|_{\infty} .
$$

But

$$
\left\|w^{h}\right\|_{\infty} \leqslant\|w\|_{\infty}+\left\|w-w^{h}\right\|_{\infty}
$$

and from (4.17),

$$
\left\|w-w^{h}\right\|_{\infty} \leqslant T^{1 / 2}\left\|\dot{w}-\dot{w}^{h}\right\|_{0} \leqslant c h^{K+1-L}\left|U_{n}-x\left(t_{n}\right)\right| .
$$

Also, from Lemma 4.7,

$$
\|w\|_{\infty} \leqslant c\|w\|_{1} \leqslant c\left|U_{n}-x\left(t_{n}\right)\right| .
$$

Thus for $h$ small enough,

$$
\left\|w^{h}\right\|_{\infty} \leqslant c\left|U_{n}-x\left(t_{n}\right)\right|
$$


Finally, from (4.16) and (4.17),

$$
\begin{aligned}
& \left\|\dot{w}^{h}+A^{T} w^{h}\right\|_{0}=\left\|\dot{w}^{h}+A^{T} w^{h}-\left(\dot{w}+A^{T} w\right)\right\|_{0} \\
& \leqslant c\left\|w^{h}-w\right\|_{1} \leqslant c h^{K+1-L}\left|U_{n}-x\left(t_{n}\right)\right| .
\end{aligned}
$$

Then (4.14) follows from (4.18), (4.19) and (4.20).

Acknowledgment. The authors wish to express their most sincere thanks to Professor Michel Fortin of the Université Laval for enlightening discussions and comments on this paper.

Centre de Recherche de Mathématiques Appliquées

Université de Montréal

C. P. 6128, Succ. A.

Montréal, Québec, Canada H3C 3J7

1. I. BABUSKKA \& J. OSBORN, "Analysis of finite element methods for second order boundary value problems using mesh dependent norms," Numer. Math., v. 34, 1980, pp. 41-62.

2. I. BabuSKKa, J. OsboRn \& J. PitKäranta, "Analysis of mixed methods using mesh dependent norms," Math. Comp., v. 35, 1980, pp. 1039-1062.

3. F. BreZzI, "On the existence, uniqueness, and approximation of saddle-point problems arising from Lagrangian multipliers," RAIRO Sér. Rouge, v. 8, 1972, pp. 129-151.

4. J. C. Butcher, "A modified multistep method for the numerical integration of ordinary differential equations,” J. Assoc. Comput. Mach., v. 12, 1965, pp. 124-135.

5. J. C. Butcher, "Coefficients for the study of Runge-Kutta integration processes," J. Austral. Math. Soc., v. 3, 1963, pp. 185-201.

6. J. C. Butcher, “Implicit Runge-Kutta processes,” Math. Comp., v. 18, 1964, pp. 50-64.

7. J. C. BUTCHER, "Integration processes based on Radau quadrature formulas," Math. Comp., v. 18, 1964, pp. 233-244.

8. J. C. Butcher, "An algebraic theory of integration methods," Math. Comp., v. 26, 1972, pp. 79-106.

9. C. CarathEodory, Vorlesungen über relle Funktionen, 2nd ed., Teubner, Leipzig, 1927.

10. P. G. Ciarlet, The Finite Element Method for Elliptic Problems, North-Holland, Amsterdam, 1978.

11. M. Crouzeix, Sur l'Approximation des Équations Différentielles Linéaires par des Méthodes de Runge-Kutta, Thèse de Doctorat d'état es-Sciences Mathématiques, Université de Paris VI, Mars 1975.

12. M. C. Delfour, "The linear quadratic optimal control problem for hereditary differential systems: Theory and numerical solution," Appl. Math. Optim., v. 3, 1977, pp. 101-162.

13. M. C. Delfour \& F. Dubeau, Piecewise Discontinuous Polynomial Approximation of Nonlinear Ordinary Differential Equations, Centre de Recherche de Mathématiques, Université de Montréal, Report \#865, 1979.

14. M. C. Delfour, W. Hager \& F. Trochu, "Discontinuous piecewise polynomial Galerkin methods for ordinary differential equations," Math. Comp., v. 36, 1981, pp. 455-473.

15. M. C. Delfour \& F. Trochu, "Discontinuous finite element methods for the approximation of optimal control problems governed by hereditary differential systems," Distributed Parameter Systems: Modelling and Identification (A. Ruberti, ed.), Springer-Verlag, New York, 1978, pp. 256-271.

16. M. C. Delfour \& F. Trochu, "Approximation des équations différentielles et problèmes de commande optimale," Ann. Sci. Math. Québec, v. 1, 1977, pp. 211-225.

17. F. Dubeau, Approximations Polynômiales par Morceaux des Équations Différentielles. Thèse de Doctorat. Université de Montréal, Février 1981.

18. M. ForTIN, Finite Element Solution of Transposed Boundary Value Problems, Proc. Second Internat. Sympos. on Innovative Numerical Analysis in Applied Engineering Sciences, University Press of Virginia, Charlotteville, Va., 1980.

19. C. W. Giar, "Hybrid methods for initial value problems in ordinary differential equations," SIAM J. Numer. Anal., v. 2, 1965, pp. 69-86.

20. W. B. (jRagG \& H. J. Stetter, “Generalized multistep predictor-corrector methods," J. Assoc. Comput. Mach., v. 11, 1964, pp. 188-209. 
21. P. C. Hammer \& J. W. Hollingsworth, "Trapezoidal methods of approximating solutions of differential equations," MTAC, v. 9, 1955, pp. 92-96.

22. B. L. Hulme, "Discrete Galerkin and related one-step methods for ordinary differential equations," Math. Comp., v. 26, 1972, pp. 881-891.

23. B. L. Hulme, “One-step piecewise polynomial Galerkin methods for initial value problems," Math. Comp., v. 26, 1972, pp. 415-426.

24. V. I. KRYLOV, Approximate Calculation of Integrals, Macmillan, New York, 1962.

25. P. Lesaint \& P. A. Raviart, "On a finite element method for solving the neutron transport equation," Mathematical Aspects of Finite Elements in Partial Differential Equations (C. de Boor, ed.), Academic Press, New York, 1974, pp. 89-124.

26. J. L. Lions \& E. Magenes, Problèmes aux Limites Non Homogènes et Applications, Vols. 1 and 2, Dunod, Paris, 1968.

27. J. C. NEDELEC, Schéma d'Approximation pour des Équations Intégro-Différentielles de Riccati, Thèse de Doctorat d'État, Paris, 1970.

28. J. T. ODEN \& L. C. Wellford, JR., "Discontinuous finite element approximations for the analysis of acceleration waves in elastic solids," The Mathematics of Finite Elements and Applications. II (J. R. Whiteman, ed.), Academic Press, London, 1976, pp. 269-284.

29. P. A. Raviart \& J. M. Thomas, "Primal hybrid finite element methods for 2 nd order elliptic equations," Math. Comp., v. 31, 1977, pp. 391-413.

30. G. Strang \& G. Fix, An Analysis of the Finite Element Method, Prentice-Hall, Englewood Cliffs, N. J., 1973.

31. J. M. Thomas, Sur l'Analyse Numérique des Méthodes d'Éléments Finis Hybrides et Mixtes, Thèse de Doctorat d’État, Université de Paris VI, 1977.

32. J. M. Thомas, "Méthodes des élements finis hybrides duaux pour les problèmes elliptiques du second ordre," RAIRO Sér. Rouge, v. 10, 1976, pp. 51-79.

33. L. C. WEllford, JR. \& T. ODEN, "Discontinuous finite-element approximations for the analysis of shock waves in nonlinearly elastic materials," J. Comput. Phys., v. 19, 1975, pp. 179-210.

34. L. C. Wellford, JR. \& J. T. Oden, "A theory of discontinuous finite element Galerkin approximations of shock waves in nonlinear elastic solids, Part I: Variational theory," Comput. Methods Appl. Mech. Engrg., v. 8, 1976, pp. 1-16.

35. L. C. Wellford, JR. \& T. OdEN, "A theory of discontinuous finite element Galerkin approximations of shock waves in nonlinear elastic solids, Part II: Accuracy and convergence," Comput. Methods Appl. Mech. Engrg., v. 8, 1976, pp. 17-36. 


\title{
Supplement to \\ Discontinuous Polynomial Approximations in the Theory of One-Step, Hybrid and Multistep Methods for Nonlinear Ordinary Differential Equations
}

\author{
By M. C. Delfour and F. Dubeau
}

APPENDIX 1.: POLYNOMIAL SPACES.

Consider the space $\mathrm{P}^{\mathrm{K}-\mathrm{L}}(0,1 ; \mathrm{E}) \times \mathrm{E}^{\mathrm{L}}$ endowed with the norm

$\|\tilde{\mathrm{w}}\|=\|\mathrm{w}\|_{0}+\sum_{\ell=1}^{\mathrm{L}}\left|w_{\ell}\right|$

where $\tilde{w}=\left(w, w_{1}, \ldots, w_{L}\right)$ and define the $L^{2}$-projector $\mathcal{P}_{L}: L^{2}(0,1 ; E) \rightarrow p^{K-L}(0,1 ; E)$ (if $L=K+1$, set $\mathrm{P}^{\mathrm{K}-\mathrm{L}}(0,1 ; E)=\{0\}$ ). Let $\left\{\tau_{\ell}\right\}_{\ell=1}^{\mathrm{L}}$ be a set of $\mathrm{L}$ distinct points outside the open interval $(0,1)$.

Lemma A1.1. The map $u \rightarrow J u: P^{K}(0,1 ; E) \rightarrow P^{K-L}(0,1 ; E) \times E^{L}$ defined by $J u=\left(\rho_{L} u, u\left(\tau_{1}\right), \ldots, u\left(\tau_{L}\right)\right)$ is an isomorphism.

Proof. We only have to show that $\mathrm{J}$ is injective. If $\mathrm{Ju}=0$, $\mathrm{u}$ has at least $L$ real roots $\left(\tau_{1}, \ldots, \tau_{L}\right)$. Thus it can be decomposed as follows: $u=\Omega \tilde{u}, \quad \Omega(\tau)=\prod_{\ell=1}\left(\tau-\tau_{\ell}\right)$,

where $\tilde{u}$ is some polynomial in $\mathrm{P}^{\mathrm{K}-\mathrm{L}}(0,1 ; \mathrm{E})$ to be determined. But $u$ belongs to $\left[\mathrm{P}^{\mathrm{K}-\mathrm{L}}(0,1 ; \mathrm{E})\right]^{\perp}$ since $P_{\mathrm{L}} \mathrm{u}=0$. As a result $\int_{0}^{1} \Omega|\tilde{u}|^{2}=\int_{0}^{1} \mathrm{u} \cdot \tilde{\mathrm{u}}=0$

and $\tilde{u}=0$ since $\Omega$ does not change sign in $[0,1]$. A fortiori $u=0$ and $J$ is

injective.

From the above result we know that there exist two strictly positive constants such that

$$
\beta_{1}\left(\tau_{1}, \ldots, \tau_{L}\right)\|u\|_{0} \leq\|J u\| \leq \beta_{2}\left(\tau_{1}, \ldots, \tau_{L}\right)\|u\|_{0} .
$$

We now show that the two constants $\beta_{1}$ and $\beta_{2}$ can be made independent of the set $\left\{\tau_{\ell}\right\}_{\ell=1}^{\mathrm{L}}$.

The hypothesis of regularity of the partition and hypothesis (3.5) suggest to consider the following compact set

$$
\mathrm{T}_{\gamma}^{\mathrm{c}}=\left\{\begin{array}{l|l}
\left(\tau_{1}, \ldots, \tau_{L}\right) \in \mathrm{R}^{\mathrm{L}}: & \begin{array}{l}
\text { (1) } \tau_{\ell} \text { is in }[\gamma, 1] \text { for all } \ell=1, \ldots, \mathrm{L} \\
\text { (2) } \tau_{\ell} \tau_{\ell-1} \geq \mathrm{c} \text { for all } \ell=2, \ldots, \mathrm{L}
\end{array}
\end{array}\right\}
$$



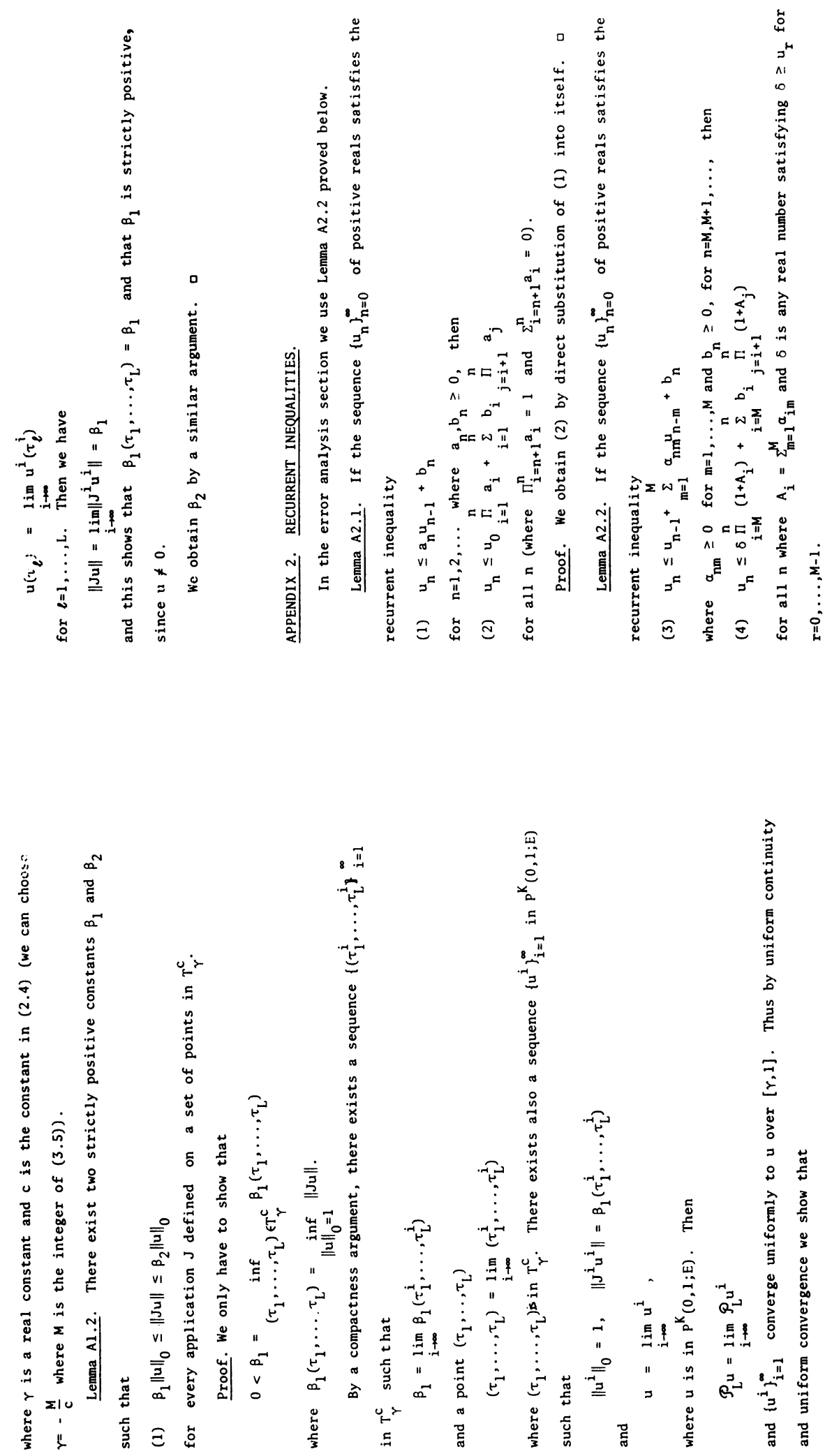

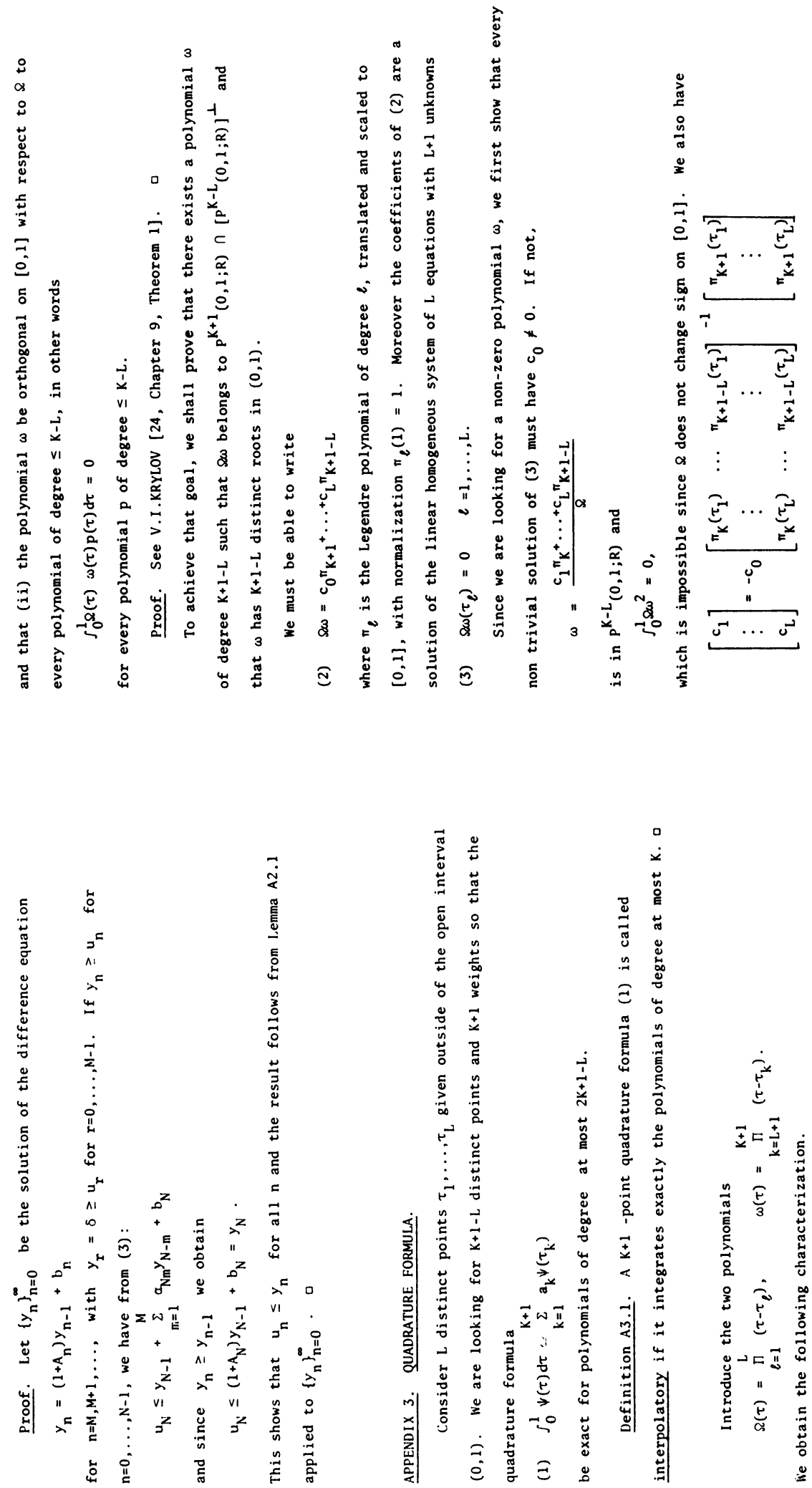

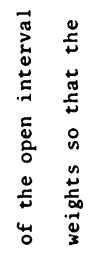

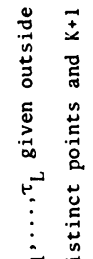

i|

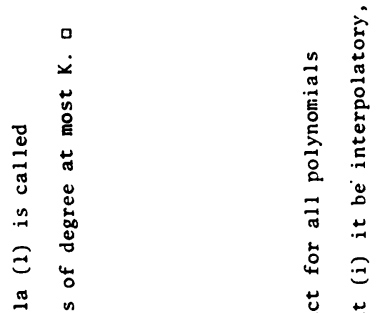

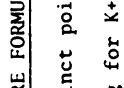

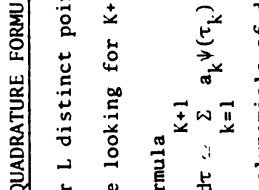

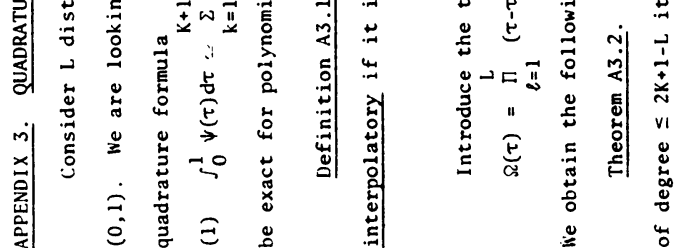



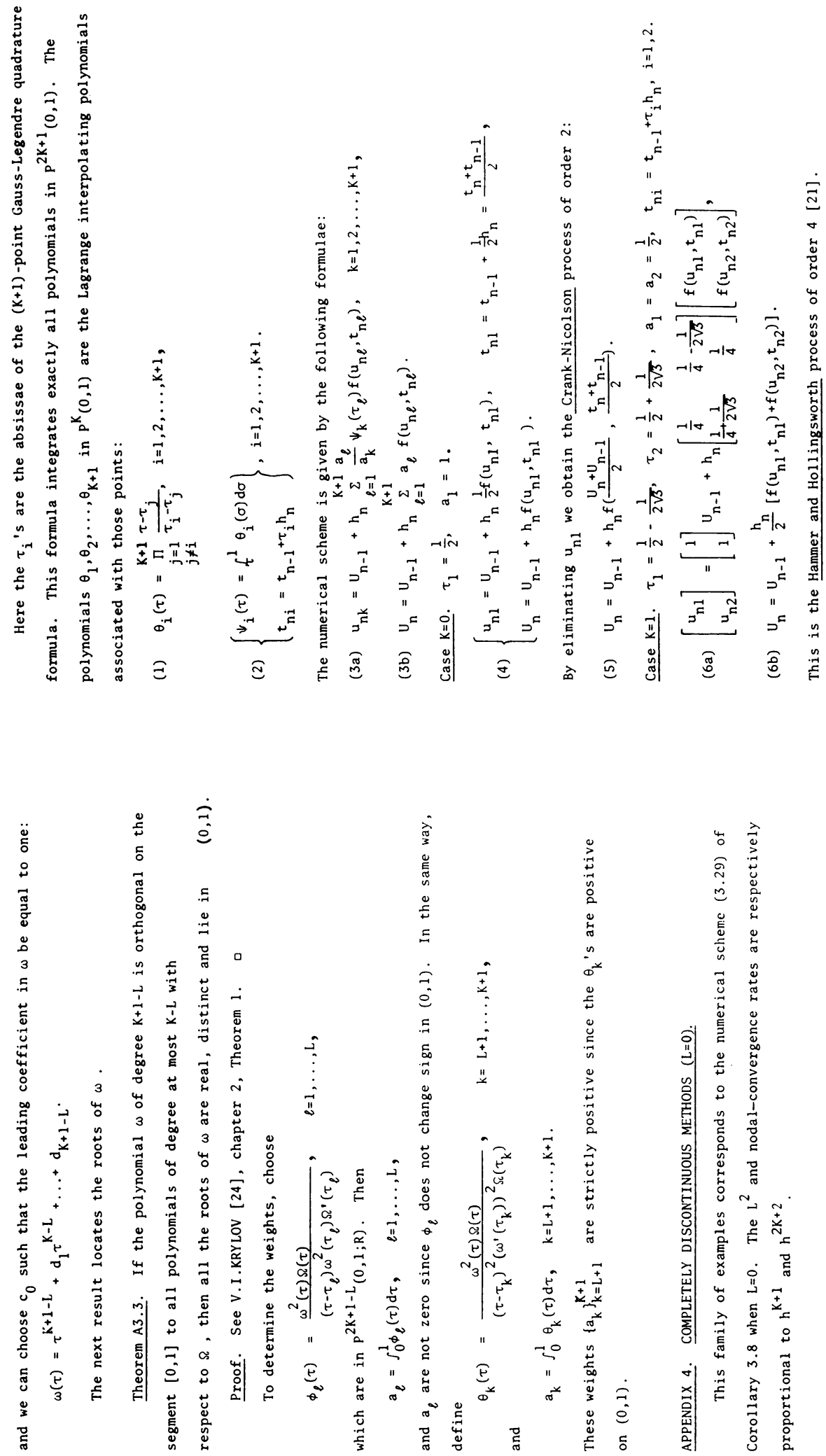

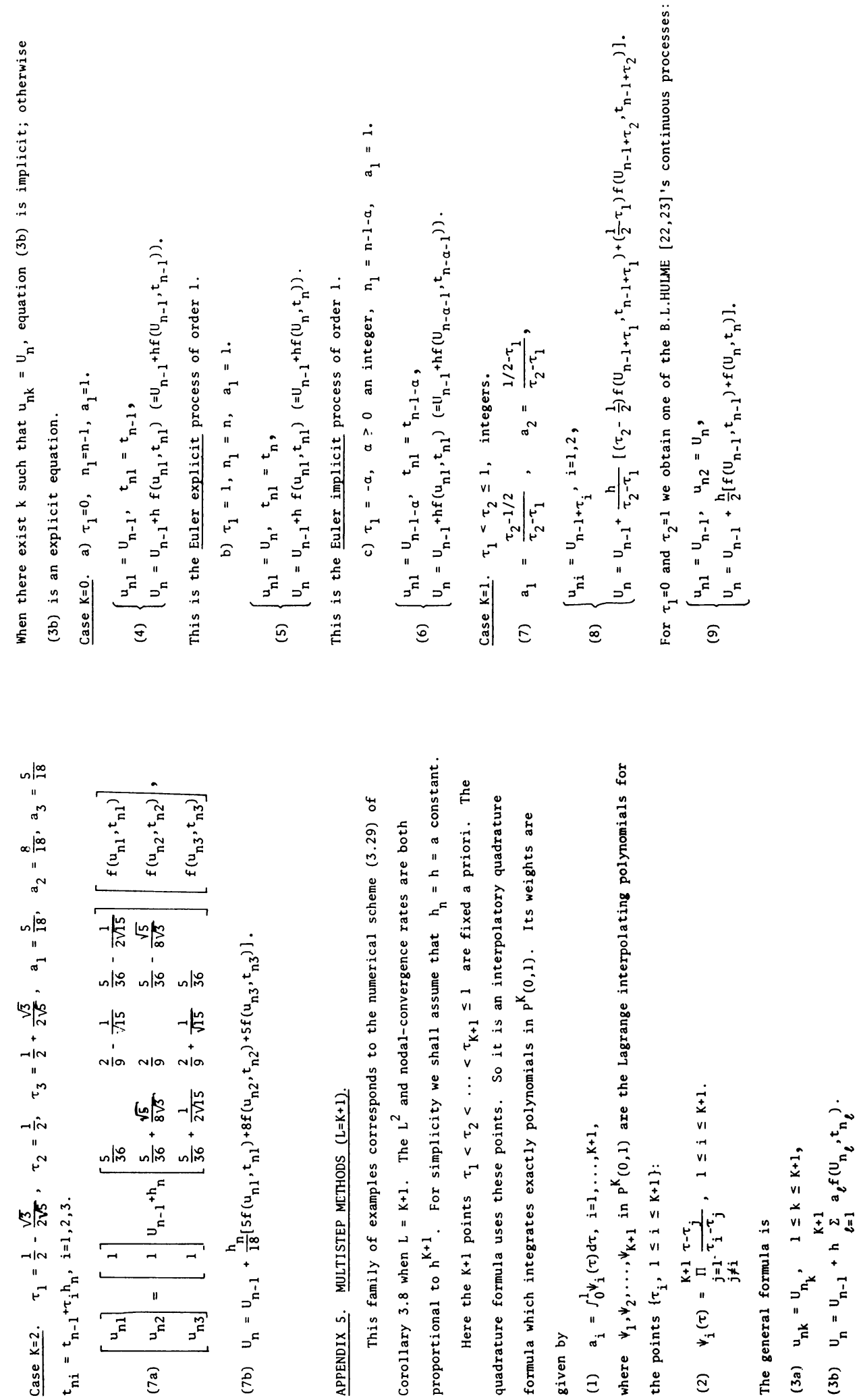

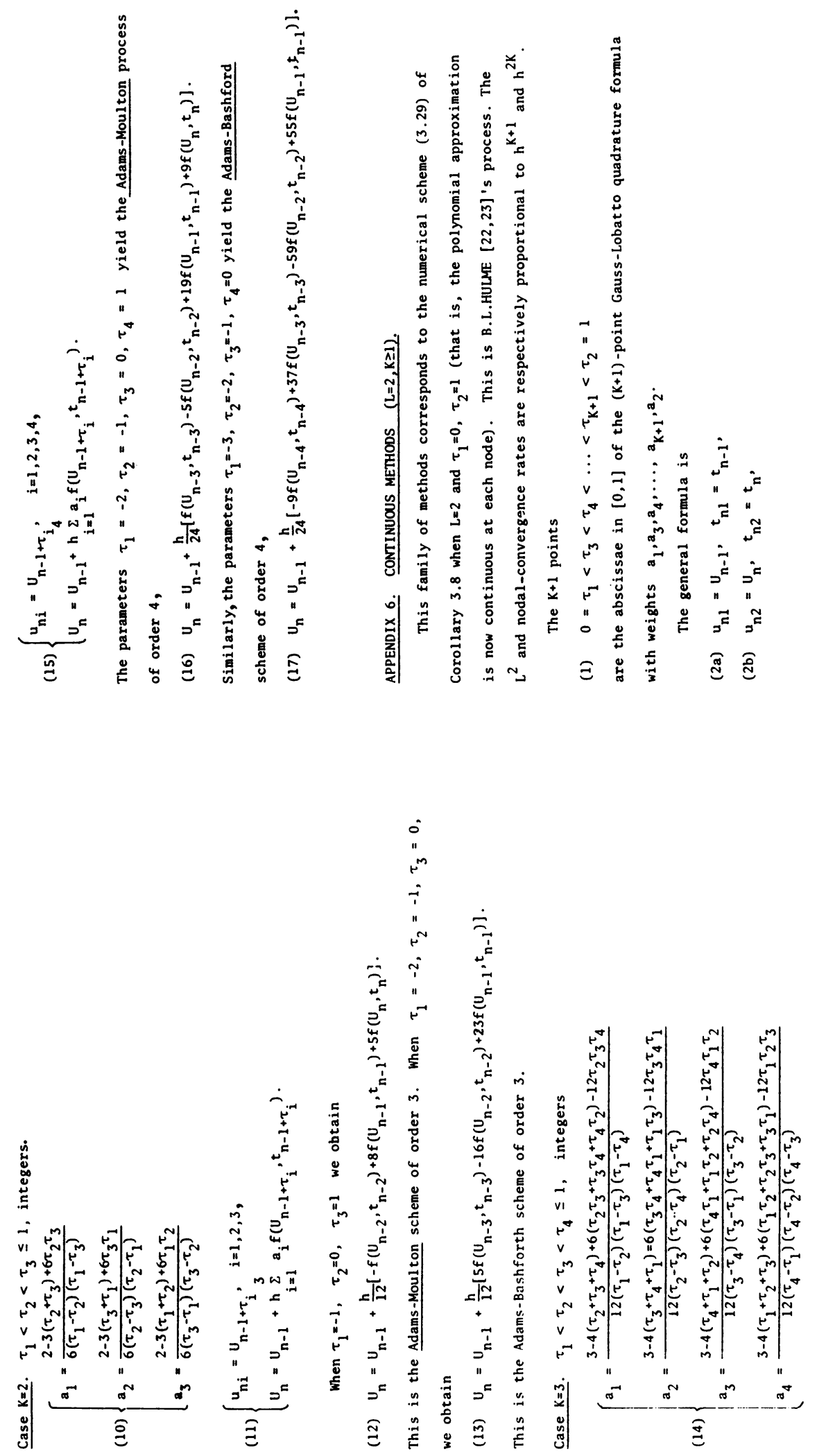

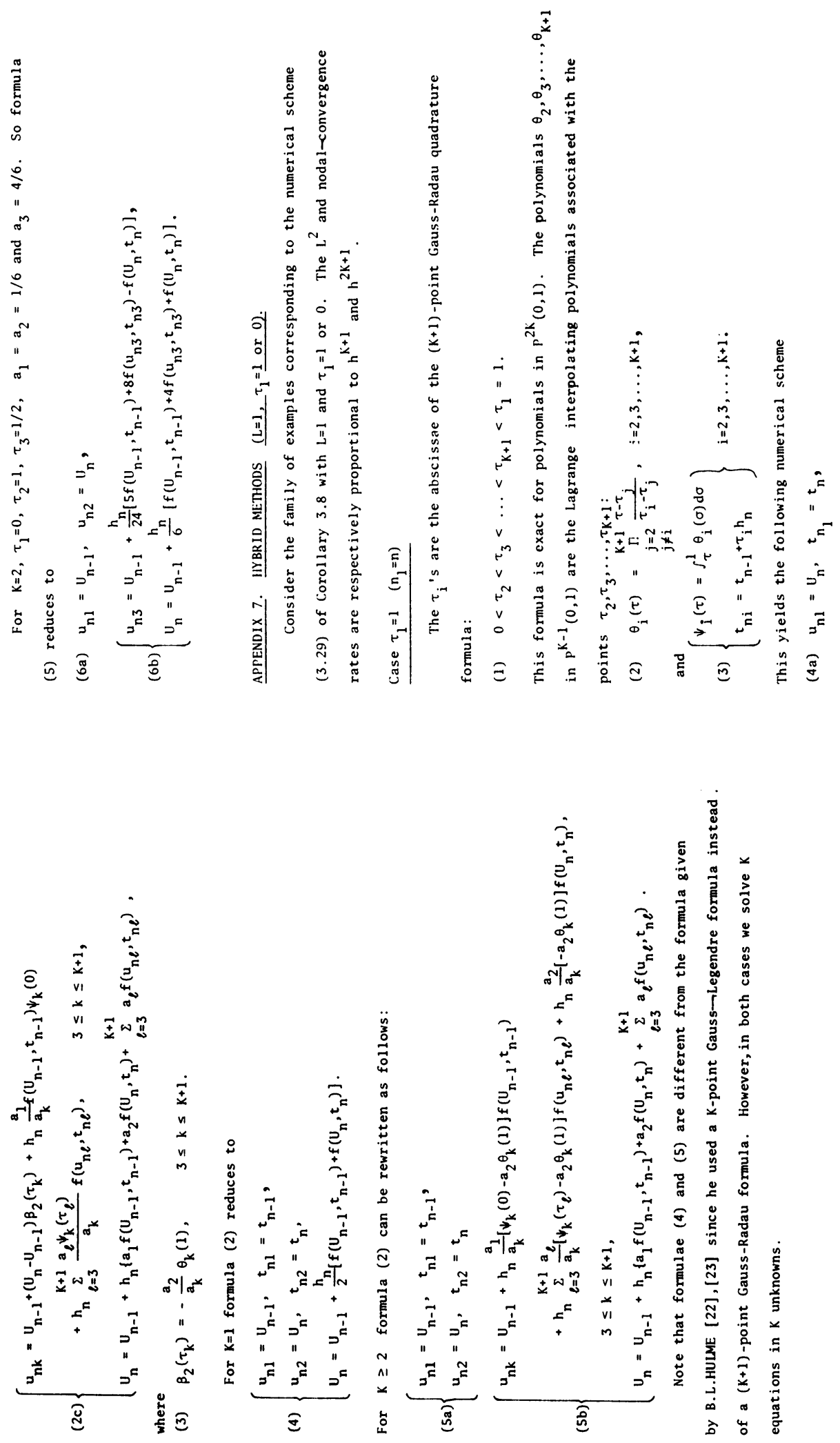

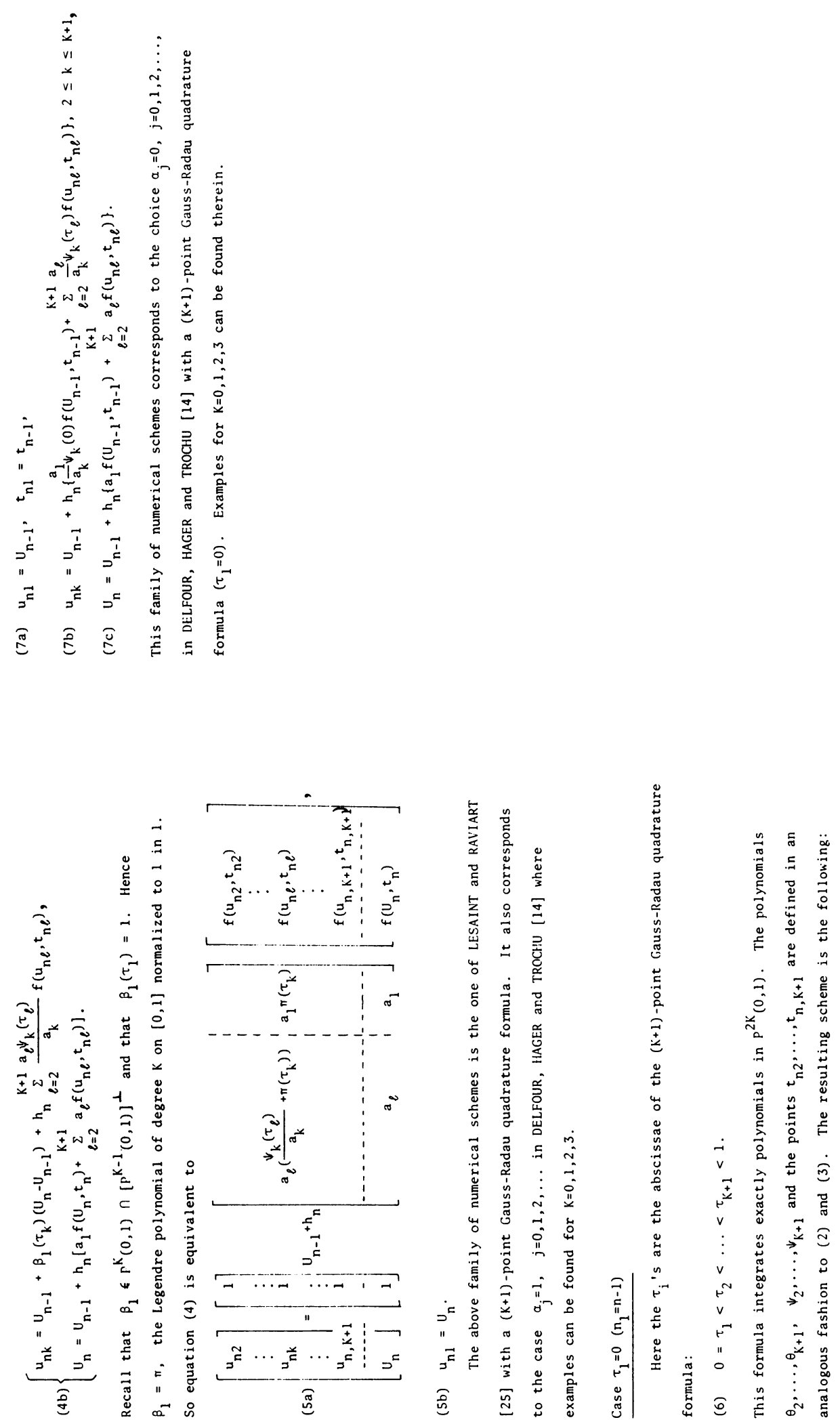